\title{
Mutational Analysis and Modeling of Negative Allosteric Modulator Binding Sites in AMPA Receptors ${ }^{\text {[s }}$
}

\author{
Charlotte Stenum-Berg, Maria Musgaard, ${ }^{1}$ Sergei Chavez-Abiega, Christine L. Thisted, \\ Lorenzo Barrella, Philip C. Biggin, and Anders S. Kristensen \\ Department of Drug Design and Pharmacology, University of Copenhagen, Copenhagen, Denmark (C.S.-B., S.C.-A., C.L.T., \\ L.B., A.S.K.); and Department of Biochemistry, University of Oxford, Oxford, United Kingdom (M.M., P.C.B.)
}

Received April 30, 2019; accepted October 1, 2019

\begin{abstract}
The $\alpha$-amino-3-hydroxy-5-methyl-4-isoxazolepropionic acid receptors (AMPARs) constitute a subclass of the ionotropic glutamate receptor superfamily, which functions as glutamategated cation channels to mediate the majority of excitatory neurotransmission in the central nervous system. AMPARs are therapeutic targets in a range of brain disorders associated with abnormal glutamate hyperactivity. Multiple classes of AMPAR inhibitors have been developed during the past decades, including competitive antagonists, ion channel blockers, and negative allosteric modulators (NAMs). At present, the NAM is the only class of AMPAR ligands that have been developed into safe and useful drugs in humans in the form of perampanel (Fycompa), which was recently approved for treatment of epilepsy. Compared with the detailed understanding of other AMPAR ligand classes, surprisingly little information has been available regarding the molecular mechanism of perampanel and other classes of NAMs at AMPARs; including the location and structure of NAM binding pockets in the receptor complex. However, structures of the AMPAR GluA2 in complex with NAMs were recently reported that unambiguously identified the NAM binding sites. In parallel with this work, our aim with the present study was to identify specific residues involved in the formation
\end{abstract}

of the NAM binding site for three prototypical AMPAR NAMs. Hence, we have performed a mutational analysis of the AMPAR region that links the four extracellular ligand-binding domains to the central ion channel in the transmembrane domain region. Furthermore, we perform computational ligand docking of the NAMs into structural models of the homomeric GluA2 receptor and optimize side chain conformations around the NAMs to model how NAMs bind in this specific site. The new insights provide potentially valuable input for structure-based drug design of new NAMs.

\section{SIGNIFICANCE STATEMENT}

The $\alpha$-amino-3-hydroxy-5-methyl-4-isoxazolepropionic acid (AMPA) receptors are glutamate-gated ion channels that mediate the majority of excitatory neurotransmission in the brain. Negative allosteric modulators of AMPA receptors are considered to have significant therapeutic potential in diseases linked to glutamate hyperactivity. The present work employs mutational analysis and molecular modeling of the binding site for prototypical NAMs to provide new molecular insight into how NAMs interact with the AMPA receptor, which is of potential use for future design of new types of NAMs.

\section{Introduction}

The $\alpha$-amino-3-hydroxy-5-methyl-4-isoxazolepropionic acid receptors (AMPARs) mediate the majority of excitatory postsynaptic currents in the central nervous system (Traynelis et al., 2010) by utilizing energy from the binding of glutamate (Glu) to drive ion channel opening. In addition to a central role

This work was supported by The Danish Council for Independent Research [grants to A.S.K.], and the Faculty of Health and Medical Science, University of Copenhagen [Ph.D. stipend to C.S.-B.]. M.M. was supported by a postdoctoral fellowship from the Alfred Benzon Foundation.

${ }^{1}$ Current affiliation: Department of Chemistry and Biomolecular Sciences, University of Ottawa, Ottawa, Canada.

https://doi.org/10.1124/mol.119.116871.

S This article has supplemental material available at molpharm. aspetjournals.org. in basal excitatory neurotransmission, AMPARs are involved in synaptic plasticity mechanisms such as long-term potentiation and depression that underlie learning and memory (Kessels and Malinow, 2009). AMPARs assemble as tetramers from combinations of GluA1-GluA4 subunits as well as a range of auxiliary subunits of different classes that can modulate receptor function (Jackson and Nicoll, 2011; Haering et al., 2014), generating a range of subtypes with overall similar core function but with differences in biophysical properties, pharmacology, and regulatory mechanisms (Cokić and Stein, 2008; Jackson and Nicoll, 2011). Structures are currently available of homomeric and heteromeric AMPARs in resting (Sobolevsky et al., 2009; Zhao et al., 2019; Herguedas et al., 2019), active (Chen et al., 2017; Twomey et al., 2017a), and

ABBREVIATIONS: Ala, alanine; AMPAR, $\alpha$-amino-3-hydroxy-5-methyl-4-isoxazolepropionic acid receptor; ATD, amino-terminal domain; blac, $\beta$-lactamase; $\left[\mathrm{Ca}^{2+}\right]_{\text {i }}$, intracellular calcium; CP (CP-465,022), 3-(2-chloro-phenyl)-2-[2-(6-diethylaminomethyl-pyridin-2-yl)-vinyl]-6-fluoro-3H-quinazolin-4-one; CTZ, cyclothiazide; DMEM, Dulbecco's modified Eagle's medium; Glu, glutamate; Gly, glycine; GYKI (GYKI-53,655), 1-(4Aminophenyl)-3-methylcarbamyl-4-methyl-3,4-dihydro-7,8-methylenedioxy-5H-2,3-benzodiazepine; HB, hydrogen bond; HEK, human embryonic kidney; LBD, ligand-binding domain; NAM, negative allosteric modulator; PAM, positive allosteric modulator; PDB, Protein Data Bank; PMP, perampanel; TEVC, two-electrode voltage clamp; TMD, transmembrane domain; WT, wild type. 
desensitized (Chen et al., 2017; Twomey et al., 2017b) states. These have advanced the molecular understanding of AMPARs by providing insight into the structural mechanisms underlying receptor function and pharmacology (Kumar and Mayer, 2013; Karakas et al., 2015; Zhu and Gouaux, 2017; Twomey and Sobolevsky, 2018).

Abnormal AMPAR activity is involved in neurologic diseases, and compounds that can modify AMPAR signaling have for decades been pursued as potential therapeutics in treatment of Alzheimer's disease, Parkinson's disease, depression, and epilepsy (Zarate and Manji, 2008; Traynelis et al., 2010; Citraro et al., 2014; Di Bonaventura et al., 2017). AMPARs contain multiple binding sites where small-molecule ligands can modulate receptor function positively or negatively via allosteric effects on core channel function (Traynelis et al., 2010; Karakas et al., 2015). This includes negative allosteric modulators (NAMs), which bind outside the orthosteric binding site or the ion channel and inhibit the receptor by noncompetitive mechanisms. Several chemical classes of AMPAR NAMs are known and include 2,3-benzodiazepines (Sólyom and Tarnawa, 2002), quinazolinones (Lazzaro et al., 2002), and pyridones (Zwart et al., 2014). Among these, the pyridone-based perampanel (PMP) under the trade name Fycompa was recently approved as a first-in-class AMPAR drug for treatment of epilepsy (Hanada, 2014), thereby demonstrating the potential of negative allosteric modulation of AMPARs in treatment of neurologic diseases linked to glutamatergic hyperfunction (Di Bonaventura et al., 2017). However, the molecular mechanisms underlying NAM inhibition of AMPARs are surprisingly poorly understood for all NAM classes; including the location and structure of the NAM binding pockets and how ligand binding can inhibit channel gating.

The AMPAR structure is highly modular and contains an extracellular amino-terminal domain (ATD) and a ligandbinding domain (LBD), a transmembrane domain (TMD) with the ion channel, and a cytoplasmic carboxyl-terminal domain (Sobolevsky et al., 2009; Kumar and Mayer, 2013). Channel gating involves allosteric coupling between LBD motions and the TMD via short flexible linkers. Binding studies have indicated that NAMs of the 2,3-benzodiazepine, quinazolinone, and pyridone classes share the same binding site (Menniti et al., 2000; Sólyom and Tarnawa, 2002; Micale et al., 2008; Mittapalli and Roberts, 2014), which is distinct from the well-characterized binding sites for positive allosteric modulators (PAMs) at the LBD interfaces (Sun et al., 2002). Early work suggested that NAM binding sites were located in the LBD-TMD linker region and identified residues in the linkers connecting the S1-M1 and S2-M4 regions to affect NAM inhibitory potency (Balannik et al., 2005; Cokić and Stein, 2008). Currently, structures of homomeric GluA2 in complex with NAMs are available that unambiguously identify each subunit containing a NAM binding pocket formed between the upper M3 transmembrane helix and the S1-M1 and S2-M4 linkers (Yelshanskaya et al., 2016). Although these structures contain electron densities for the bound NAM ligands, the resolution of approximately $4 \AA$ does not allow direct identification of NAM-protein interactions (Twomey and Sobolevsky, 2018).

We have conducted a mutational analysis of the LBD-TMD linker regions in the GluA2 subunit to further study the role of LBD-TMD linker residues for the function of three prototypical NAMs in the form of PMP, 1-(4-Aminophenyl)-3methylcarbamyl-4-methyl-3,4-dihydro-7,8-methylenedioxy5H-2,3-benzodiazepine [GYKI-53,655 (GYKI)] (Moncada et al., 1991), and quinazolinone 3-(2-chloro-phenyl)-2-[2-(6diethylaminomethyl-pyridin-2-yl)-vinyl]-6-fluoro- $3 H$-quinazolin4-one [CP-465,022 (CP)] (Menniti et al., 2000). Our initial aim was to use the resulting data set to specify the location of NAM binding pockets in the linker region and employ computational ligand docking of NAMs into GluA2 structures to create binding mode models. We used the new GluA2/NAM structures as templates to create binding models that include all side chains. In combination with our mutational analysis, the models provide molecular insight into ligand orientations and specific protein-ligand contacts for the investigated NAMs.

\section{Materials and Methods}

Materials. All chemicals were obtained from Sigma-Aldrich (St. Louis, MO) unless otherwise stated. Dulbecco's modified Eagle's medium (DMEM), FBS, trypsin, and penicillin-streptomycin were obtained from Invitrogen (Carlsbad, CA). DNA-modifying enzymes were obtained from New England Biolabs (Ipswich, MA), except PfuUltra II Fusion HS DNA Polymerase (Agilent, Carlsbad, CA) and Rapid T4 Ligase (Roche Molecular Systems, Pleasanton, CA). All tissue cell culture plasticware were obtained from Sarstedt (Nümbrecht, Germany) unless otherwise stated. 6-Cyano-7-nitroquinoxaline-2,3-dione, cyclothiazide (CTZ), and CP-465,022 hydrochloride were obtained from HelloBio (Bristol, UK). GYKI-53,655 or GYKI53,655 hydrochloride was obtained from Axon MedChem (Groningen, Netherlands) or Tocris Bioscience (Bristol, UK). Perampanel was obtained from Toronto Research Chemicals (Toronto, Ontario, Canada) or Apexmol (Beijing, China). The calcium dyes Fluo-2 or Fluo-8 AM were obtained from TEFlabs (Austin, TX) or AAT Bioquest (Sunnyvale, CA).

Molecular Biology. The plasmid vectors pRK5 (BD PharMingen, San Diego), pGEM-HE (Liman et al., 1992) and pXOOF containing cDNA for the unedited flip isoform of the rat GluA2 gene were used for expression in mammalian cells or generation of mRNA for microinjection in Xenopus laevis oocytes. The pXOOF vector is a modified version of the dual-purpose mammalian and Xenopus expression vector pXOON (Jespersen et al., 2002), in which cDNA encoding enhanced GFP is removed. Specifically, we created pXOOF by polymerase chain reaction amplification of the entire $\mathrm{pXOON}$ sequence excluding the enhanced GFP gene by using forward and reverse primers with $5^{\prime}$ overhang recognition sequence for the restriction enzyme $S b f \mathrm{I}$. The resulting linear DNA fragment was $S b f \mathrm{I}$ digested and religated using T4 DNA Ligase (Roche, Mannheim, Germany) to generate $\mathrm{pXOOF}$. The $\mathrm{pXOOF}$ plasmid sequence was verified by DNA sequencing. Generation of GluA2 point mutants was performed by site-directed mutagenesis using the QuickChange Mutagenesis Kit (Stratagene, La Jolla, CA). The mutations were verified by DNA sequencing of the entire GluA2 gene (GATC Biotech, Constance, Germany). For analysis of cell-surface expression of wild-type (WT) and mutant GluA2 receptors, cDNA encoding $\beta$-lactamase (blac) was inserted in the GluA2 cDNA in between the segments encoding the $N$-terminal signal sequence and the ATD. Specifically, using In-Fusion cloning (Mountain View, CA), a polymerase chain reaction-amplified DNA fragment encoding blac flanked by two short amino acid linkers (GGSGS and GGSG) was inserted in-frame into an AfeI site introduced by site-directed mutagenesis of codons 26 and 27 in the GluA2 sequence to create blac-tagged GluA2. For use as a template for in vitro transcription of complementary RNA, pGEM$\mathrm{HE}$ and pXOOF plasmid constructs were linearized downstream from the 3 '-untranslated region of the Xenopus $\beta$-globin sequence using the NheI-HF restriction enzyme, and purified by ethanol precipitation 
with Pellet Paint NF Co-Precipitant (Merck, Billerica, MA). Complementary RNA transcription was performed using the AmpliCap-Max T7 High Yield Message Maker Kit (CellScript, Madison, WI) and RNA was column purified using the NucleoSpin RNA Clean-up XS Kit (Macherey-Nagel, Düren, Germany).

Mammalian Cell Culturing and Expression. Human embryonic kidney (HEK) 293T cells (American Type Culture Collection, Manassas, VA) were cultured in growth medium (DMEM supplemented with $10 \% \mathrm{v} / \mathrm{v}$ FBS, $100 \mathrm{U} / \mathrm{ml}$ penicillin, and $100 \mu \mathrm{g} / \mathrm{ml}$ streptomycin) at $37^{\circ} \mathrm{C}$ in a humidified $5 \% \mathrm{CO}_{2}$ environment. For expression of WT and mutant GluA2 in HEK293T cells, TransIT-LT1 DNA Transfection Reagent (Mirus, Madison, WI) was used as described previously (Sørensen et al., 2014). Briefly, HEK293T cells in suspension $\left(1 \times 10^{6}\right.$ cells $\left./ \mathrm{ml}\right)$ were mixed with $\mathrm{DNA} /$ transfection complex (formed by mixing plasmid DNA, TransIT-LT1 reagent, and DMEM in a 1:3:90 ratio) and plated into poly-D-lysine-coated Falcon black clear-bottom 96-well plates (Corning, Corning, NY) to a final density of approximately 20,000 cells and $0.1 \mu \mathrm{g}$ plasmid DNA per well. The competitive antagonist 6-cyano-7-nitroquinoxaline-2,3dione was added at a final concentration of $20 \mu \mathrm{M}$ to protect against glutamate-induced cytotoxicity in the transfected cultures. Cells were incubated for 2 days after transfection before experiments.

Xenopus Laevis Oocyte Expression. Defollicated Xenopus oocytes (stages V to VI) were prepared and injected with 1-25 ng mRNA, as described previously (Poulsen et al., 2013). The care and use of Xenopus laevis were in strict adherence to a protocol approved by the Danish Veterinary and Food Administration (license 2014-150201-00031). Injected oocytes were incubated at $18^{\circ} \mathrm{C}$ in standard Barth's solution containing $88 \mathrm{mM} \mathrm{NaCl}, 1 \mathrm{mM} \mathrm{KCl}, 0.41 \mathrm{mM} \mathrm{CaCl} \mathrm{Cl}_{2}$, $2.4 \mathrm{mM} \mathrm{NaHCO}_{3}, 0.33 \mathrm{mM} \mathrm{Ca}\left(\mathrm{NO}_{3}\right)_{2}, 0.82 \mathrm{mM} \mathrm{MgSO}_{4}$, and $5 \mathrm{mM}$ Tris (pH 7.4), supplemented with $50 \mu \mathrm{g} / \mathrm{ml}$ gentamycin and used for twoelectrode voltage-clamp electrophysiology (TEVC) measurements 27 days after injection.

Intracellular $\mathbf{C a}^{2+}$ Imaging Assay. For determination of concentration-response curves for glutamate activation and NAM inhibition of WT and mutant GluA2 receptors, imaging of changes in the concentration of intracellular $\mathrm{Ca}^{2+}$ in transfected HEK293T cells was performed using a FlexStation I Plate Reader (Molecular Devices, Sunnyvale, CA) to measure changes in the fluorescence of a calciumsensitive fluorescent dye in 96 -well plates. On the day of experiments, transfected cells were washed three times in PBS supplemented with $\mathrm{CaCl}_{2}$ and $\mathrm{MgCl}_{2}$ [137 $\mathrm{mM} \mathrm{NaCl}, 2.7 \mathrm{mM} \mathrm{KCl}, 10 \mathrm{mM} \mathrm{Na}_{2} \mathrm{HPO}_{4}, 2 \mathrm{mM}$ $\mathrm{KH}_{2} \mathrm{PO}_{4}, 0.1 \mathrm{mM} \mathrm{CaCl}_{2}$, and $0.5 \mathrm{mM} \mathrm{MgCl}_{2}$ (pH 7.4)] and loaded with a solution containing $2 \mu \mathrm{M}$ Fluo-2 or Fluo-8 AM fluorescent indicator dye (dissolved in plain DMEM and incubated for 30 minutes at $37^{\circ} \mathrm{C}$ ). Excess loading dye was removed by washing three times in FLUO buffer containing $140 \mathrm{mM}$ choline chloride, $5 \mathrm{mM} \mathrm{KCl}, 1 \mathrm{mM} \mathrm{MgCl}{ }_{2}$, $10 \mathrm{mM} \mathrm{CaCl}_{2}$, and $10 \mathrm{mM}$ HEPES (pH 7.4). For NAM IC 50 experiments, cells were preincubated with $50 \mu \mathrm{l}$ FLUO buffer containing various concentrations of NAM for 10 minutes at room temperature to achieve equilibrium before measurement of agonist responses. Maximum final NAM concentrations were $100 \mu \mathrm{M}$ PMP, $100 \mu \mathrm{M}$ CP465,022 , and $2 \mathrm{mM}$ GYKI-53,655. The highest final DMSO concentration was $4 \%(\mathrm{v} / \mathrm{v})$ for GYKI-53,655 and $0.2 \%(\mathrm{v} / \mathrm{v})$ for perampanel and CP-465,022. DMSO concentrations up to $4 \% \mathrm{v} / \mathrm{v}$ had minimal effect on the glutamate responses in HEK293T cells (data not shown). Changes in dye fluorescence in response to the addition of agonist solution were then measured at $538 \mathrm{~nm}$ using excitation at $485 \mathrm{~nm}$ and emission cutoff at $515 \mathrm{~nm}$. Baseline fluorescence was measured for 18 seconds before the addition of $50 \mu \mathrm{l}$ agonist solution to each well of the assay plate, and fluorescence was measured for 72 seconds after addition of the agonists. Peak fluorescence was calculated as the difference between maximal observed increase in fluorescence and preagonist baseline fluorescence. Agonist solutions were prepared in FLUO buffer in clear V-bottom 96-well plates (Thermo Fischer Scientific, Waltham, MA), which contained $100 \mu \mathrm{M} \mathrm{CTZ}$ and $600 \mu \mathrm{M}$ glutamate unless otherwise stated. Generally, experiments were performed in quadruplicate wells for each mutant and compound concentration and repeated on three independent days. Preincubation with NAMs in some instances produced concentration-dependent inhibition of the baseline measurement before Glu addition, which resulted in an apparent increase in response amplitude with increasing NAM concentration. This effect likely arises from activation by small amounts of contaminating Glu produced by the cells that were not removed by thorough washing. The observation might indicate an effect of the desensitization state of the receptor on NAM potency.

Determination of Cell-Surface Expression. For determination of mutational effects on cell surface expression of GluA2 receptors, mutants were created in a GluA2 subunit construct tagged with a bacterial blac enzyme. Relative levels of surface-expressed blactagged GluA2 (i.e., blac-GluA2) can be accurately quantified in living cells by measuring the conversion rate of the membrane-impermeable substrate nitrocefin by simple absorption spectroscopy (Lam et al., 2013). For analysis in HEK293T cells, transfected cells were cultured in poly-D-lysine-coated clear-bottom 96-well plates for 2 days. Cells were washed twice in PBS supplemented with $\mathrm{CaCl}_{2}$ and $\mathrm{MgCl}_{2}$, followed by addition of nitrocefin to a final concentration of $50 \mu \mathrm{M}$ in a total volume of $100 \mu \mathrm{l}$ per well. Immediately following nitrocefin addition, plates were placed in a microplate reader (Safire2; Tecan, Maennedorf, Switzerland), and well absorbance at $486 \mathrm{~nm}$ of the nitrocefin conversion product was recorded every minute for 60 minutes at $\sim 30^{\circ} \mathrm{C}$. For each well, the absorbance at $486 \mathrm{~nm}$ was plotted as a function of time using GraphPad Prism version 6.01 (GraphPad Software, San Diego, CA) and the rate of nitrocefin conversion per minute was determined by linear regression analysis of the slope of the curve in the linear range.

TEVC Electrophysiology. For TEVC recordings, glass micropipettes (0.69 mm i.d/1.2 mm o.d.; Harvard apparatus, Holliston, MA) were pulled on a Sutter P-1000 to a tip resistance of 0.5-2.5 M $\Omega$ and filled with $3 \mathrm{M} \mathrm{KCl}$. Oocytes were clamped using a TEVC amplifier (OC-725C; Warner Instruments, Hamden, CT) and continuously perfused with frog Ringer's solution containing $115 \mathrm{mM} \mathrm{NaCl}, 2 \mathrm{mM}$ $\mathrm{KCl}, 5 \mathrm{mM}$ HEPES, and $1.8 \mathrm{mM} \mathrm{BaCl}_{2}$ (pH 7.6 adjusted with $\mathrm{NaOH}$ ), via the force of gravity at flow rates of $5-10 \mathrm{ml} / \mathrm{min}$ into a vertical oocyte flow chamber with a volume of $0.3 \mathrm{ml}$ (Joshi et al., 2004). Compounds dissolved in frog Ringer's solution were added by bath application. This perfusion system yields a solution exchange time of 100-500 milliseconds. Concentration-response data were generally recorded at a holding potential of -30 or $-60 \mathrm{mV}$, and each compound solution was applied for 30 or 45 seconds to ensure that steady-state currents were obtained. NAM concentration-response curves were obtained by adding increasing concentrations of NAM in the continuous presence of $300 \mu \mathrm{M}$ glutamate after evoking a full agonist response by adding $300 \mu \mathrm{M}$ glutamate alone. All experiments were performed at room temperature $\left(20-23^{\circ} \mathrm{C}\right)$. Data acquisition was accomplished using an analog-digital converter (CED 1401 Plus; Cambridge Electronic Design, Cambridge, UK) interfaced with a PC running WinWCP software (available from Strathclyde Electrophysiology Software, University of Strathclyde, Glasgow, UK). Concentration-response and concentration-inhibition measurements were performed by measuring agonist-evoked current during stepwise application of increasing concentrations of agonist or NAM (as illustrated in Fig. 6).

Patch-Clamp Electrophysiology (HEK Cells). For electrophysiological experiments involving rapid ligand-application measurements, outside-out membrane patches were excised from transiently transfected HEK293 cells using thin-wall glass micropipettes (TW150F-4; World Precision Instruments), heat polished to 3-10 M $\Omega$ tip resistance filled with internal solution containing $140 \mathrm{mM} \mathrm{KCl}, 10 \mathrm{mM}$ HEPES, and $1 \mathrm{mM}$ 1,2-bis(o-aminophenoxy) ethane- $N, N, N^{\prime}, N^{\prime}$-tetraacetic acid (BAPTA) (pH 7.20, adjusted with $\mathrm{KOH})$ and osmolality adjusted to $285 \mathrm{mOsm}$ by adding $10 \mathrm{mM}$ D-Glucose. Pipettes had a tip resistance of 3-10 M $\Omega$. External recording solution for all experiments was comprised of $150 \mathrm{mM} \mathrm{NaCl}$, $3 \mathrm{mM} \mathrm{KCl}, 10 \mathrm{mM}$ HEPES, $0.01 \mathrm{mM}$ EDTA, and $1 \mathrm{mM} \mathrm{CaCl}_{2}$ (pH 7.40) with $\mathrm{NaOH}(290 \mathrm{mOsm})$. Currents were recorded at a holding 
potential of $-60 \mathrm{mV}$ with an Axopatch 200B amplifier (Molecular Devices), low-pass filtered at $8 \mathrm{kHz}$ using an 8-pole Bessel filter (Frequency Devices, Ottawa, IL), and digitized at $40 \mathrm{kHz}$ using a Digidata 1440A (Molecular Devices). Rapid application and removal of ligand solutions at excised membrane patches were performed using a piezo (Burleigh Instruments, Fishers, NY) bimorph-driven double-barreled theta tube perfusion system. The time course of solution exchange across the laminar flow interface was estimated by liquid junction potential measurements of $0.2-0.4$ milliseconds (10\%-90\% rise time) for a 10 -fold difference in ionic strength. Current kinetics results were analyzed using ChannelLab version 2 (Synaptosoft, Decatur, GA).

Data and Statistical Analyses. For construction of concentrationresponse curves from intracellular calcium $\left(\left[\mathrm{Ca}^{2+}\right]_{\mathrm{i}}\right)$ imaging experiments, peak fluorescence response (corresponding to the difference between the maximum and baseline $538 \mathrm{~nm}$ emission) from quadruplicate wells representing identical NAM or agonist concentrations were averaged using SoftMax Pro version 5.4 software (Molecular Devices). For each individual plate experiment, the average responses were normalized to the average corresponding maximal agonist response or the agonist response in absence of NAM. Composite concentration-response curves were generated by plotting normalized data from multiple experiments as a function of NAM or agonist concentration using GraphPad Prism software. Concentration-response relationships were fitted as composite curves using GraphPad Prism version 6.01 (GraphPad Software) to the Hill equation:

$$
\text { response }=\text { bottom }+\frac{\text { top }- \text { bottom }}{1+10^{\left(\log X C_{50}-X\right) \cdot n H}}
$$

where bottom is the fitted minimum response; top is the fitted maximum response, $\mathrm{nH}$ is the Hill slope; $X$ is the concentration; and $X C_{50}$ is the $\mathrm{IC}_{50}$ (half-maximally effective concentration of inhibitor) or $\mathrm{EC}_{50}$ (half-maximally effective concentration of agonist), respectively. For the construction of concentration-response curves from electrophysiological data, agonist-evoked current responses from individual oocytes were determined from TEVC traces using ClampFit version 10.3 (Molecular Devices) and normalized to the current response obtained in the absence of NAM (for IC $_{50}$ determinations) or by maximal agonist concentration (for $\mathrm{EC}_{50}$ determinations). Concentration-response curves were constructed as composites of normalized responses from three to six oocytes and analyzed as described for the $\left[\mathrm{Ca}^{2+}\right]_{\mathrm{i}}$ assay. Unless otherwise noted, results are expressed as mean \pm S.D. Statistical analysis of pairwise and multiple comparisons was performed using Student's $t$ test or ANOVA with multiple comparisons procedures as indicated.

Molecular Modeling. The crystallographic structures of the GluA2 receptor used as input models for ligand docking included the apo state structure [Protein Data Bank (PDB) 5L1B], the GYKI-bound structure (PDB 5L1H), the CP-bound structure (PDB 5L1E), and the PMP-bound structure (PDB 5L1F). All structures were determined by Yelshanskaya et al. (2016) and obtained from the Brookhaven Protein Data Bank (Berman et al., 2000). All four structures have several missing residues and residues with missing side chain atoms. For the initial docking calculations, missing atoms in locations that were distant from the NAM binding sites (located at the extracellular side of the TMD) were not modeled; this included residues located in the ATD-LBD linker region, the M1-M2 linker region, and the C-terminal region. Within and around the NAM binding pockets, positions with missing atoms are located in the S1-M1 linker region, the M3 transmembrane helix, the M3-S2 linker, and the S2-M4 linker. For these regions the missing atoms were added using the WHAT-IF webserver interface (Vriend, 1990), following the method described by Chinea et al. (1995). The ligand molecules cocrystallized with the GluA2 receptor in PDB files $5 \mathrm{~L} 1 \mathrm{H}, 5 \mathrm{~L} 1 \mathrm{E}$, and $5 \mathrm{~L} 1 \mathrm{~F}$ were imported into the Maestro software (Schrödinger Release 2013-2: Maestro, version 9.5; Schrödinger, LLC, New York), where hydrogen atoms were added. The ligands were either left in the crystal conformation or subjected to short energy minimization using the clean-up geometry function; giving rise to two conformations for each imported structure. The original ligand structures (with hydrogen atoms added) and the equivalent energy-minimized structures were used as input for docking calculations (i.e., in total, eight structures per ligand from the four different subunits; each in either original or optimized conformation). Additionally, for each ligand two conformations were generated by constructing the ligand from scratch in Maestro, meaning that 10 input ligand structures were used for docking of each of the ligands CP, PMP, and GYKI. It should be noted that PMP, $\mathrm{CP}$, and GYKI have low solubility in water, suggesting that they are neutral at physiologic $\mathrm{pH}$; therefore, they were docked in their unprotonated states. All ligand dockings into the GluA2 receptor structures were performed using the Autodock Vina docking software (Trott and Olson, 2010), utilizing a search box that covered all four binding pockets. Twenty poses were saved in each docking run during which the ligands were treated flexibly, while the protein remained rigid. In general, the poses showed variations in the position of the ligand due to the relatively large search box. However, the binding pocket was identified for all three ligands when docked into their parent protein structure and assumed orientations similar to those proposed by the existing GluA2/NAM structures (Yelshanskaya et al., 2016). Based on the assumption that these ligand orientations are overall correct, we constructed up to 100 models for each compound (98 for GluA2:GYKI and 99 for GluA2:PMP and GluA2:CP), i.e., generating approximately 400 binding sites since the NAM:GluA2 stoichiometry (at least in the crystal structures) is 4:1. In this alternative approach, different side chain conformations were sampled around the ligand-binding sites using modeling software MODELLER (Sali and Blundell, 1993) to build models of the GluA2/NAM complex for which all missing atoms and residues were added. Furthermore, all residue positions within $12 \AA$ of the ligands were optimized. MODELLER can accommodate the ligands in terms of space; however, without parameters for the ligands, the interactions with the protein atoms of the binding site are not included in the optimization. Thus, to account for protein-ligand interactions and to optimize the ligand conformations, all of the generated models were subjected to energy minimization in GROMACS 5.0 (Van Der Spoel et al., 2005) using the AMBER99-ILDN (Lindorff-Larsen et al., 2010) force field for the protein and ligand parameters from the general AMBER force field (Wang et al., 2004) generated using Antechamber in AmberTools14 (Salomon-Ferrer et al., 2013). Acpype (Sousa da Silva and Vranken, 2012) was used to generate GROMACS topologies from the AMBER ones. Minimizations were performed in vacuum until the maximum force on a single atom was less than $100 \mathrm{~kJ} / \mathrm{mol}$ per nanometer or machine precision was reached. Analysis and image generation were performed using the Maestro software along with VMD software (Humphrey et al., 1996) and PyMOL software.

\section{Results}

Glycine-Scanning Mutagenesis of the S1-M1 and S2-M4 Linker Regions. Previous mutational analysis had identified the LBD-TMD linker segments as overall candidate regions to form the NAM binding sites in the AMPAR complex (Balannik et al., 2005). Specifically, mutation of residues in the S1-M1 and S2-M4 segments can perturb NAM inhibition, and these residues may thus be involved in forming NAM binding pockets as well as interacting directly with NAMs (Fig. 1). However, the NAM binding site likely involves additional residues in the linker segments as well as upper M1 and M4 segments. To expand the knowledge of the contribution of the side chains of individual residues in the S1-M1 (including the pre-M1 helix), upper M1, S2-M4, and upper M4 segments for NAM binding, we initially performed glycine (Gly) scanning mutagenesis of all amino acid positions 
between K511 and M527 in the S1-M1 and upper M1 segments and E782 and Y797 in the S2-M4 and upper M4 segments (Fig. 3). We selected the flip isoform of the GluA2 subunit in the unedited form as the model AMPAR subunit for the mutational study based on the robust expression and wellcharacterized functional properties of homomeric GluA2 receptors. In addition, several X-ray crystallographic and cryo-electron microscopy structures exist for homomeric GluA2 receptors (Sobolevsky et al., 2009; Yelshanskaya et al., 2014; Twomey et al., 2016, 2017b), which provide an excellent framework for interpreting mutagenesis results in a structural context and can serve as target structures for ligand docking. Notably, the target LBD-TMD linker regions are conserved between subunits GluA1-4. Thus, if the NAM binding sites are harbored in the linker region, homo- or heteromeric AMPARs formed of any subunit combination may potentially be equivalent with regard to the structure of the NAM binding pocket. To allow determination of mutational effects on surface expression, we used a version of GluA2 tagged in the $N$-terminal of the ATD with the reporter enzyme blac (blac-GluA2; see Materials and Methods) as the parent construct for all mutants. To ensure that fusion of blac to the ATD does not change the function of the GluA2 receptor, we compared current responses from WT GluA2 and blac-GluA2 in excised outside-out membrane patches from HEK293 cells to the application of a maximally effective concentration $(10 \mathrm{mM})$ of glutamate by using a rapid perfusion system with a solution exchange time $<0.4$ milliseconds (see Materials and Methods) (Supplemental Fig. 1); the results showed that the rates of receptor activation, desensitization, and deactivation were similar with and without blac fused to the ATD (Supplemental Fig. 1). Furthermore, we determined the $\mathrm{IC}_{50}$ values for NAM inhibition of WT GluA2 and blac-GluA2 currents in Xenopus oocytes (see Materials and Methods) and did not observe any significant effect of blac on NAM pharmacology (Supplemental Fig. 1; Tables 1 and 2).

For initial characterization of the mutant library, we expressed mutants individually in HEK293 cells and used an intracellular calcium concentration imaging assay to measure concentration-dependent Glu-evoked increases in $\left[\mathrm{Ca}^{2+}\right]_{\mathrm{i}}$ (see Materials and Methods) and assessed the impact of Gly substitutions on basal receptor function by determining the $\mathrm{EC}_{50}$ and maximum response amplitude $\left(E_{\max }\right)$ for Glu (Fig. 2). Also, we measured blac activity to determine mutational effects of the ability of the mutant GluA2 subunits to correctly fold, assemble, and traffic to the cell surface (Supplemental Fig. 2; Supplemental Table 1). The results of the Gly scanning mutagenesis are summarized in Fig. 3 and Table 1. For the 16 positions in the S1-M1 and upper M1 segments, Gly substitution (which removes the WT residue side chain) appeared to disrupt receptor function at five positions (L518, P520, L521, I525, and W526) since these mutants, when expressed in HEK293 cells, did not produce clearly detectable $\mathrm{Ca}^{2+}$ signals in the $\left[\mathrm{Ca}^{2+}\right]_{\mathrm{i}}$ imaging assay in response to Glu concentrations up to $1 \mathrm{mM}$ (for a representative example, see Fig. 2A). However, expression of all five mutants generated extracellular blac activity in HEK293 cells that were within the range of the activity observed for expression of WT blac-GluA2 receptors (Supplemental Table 1), indicating that these Gly substitutions maintain the ability of the GluA2 subunit to fold and traffic to the cell

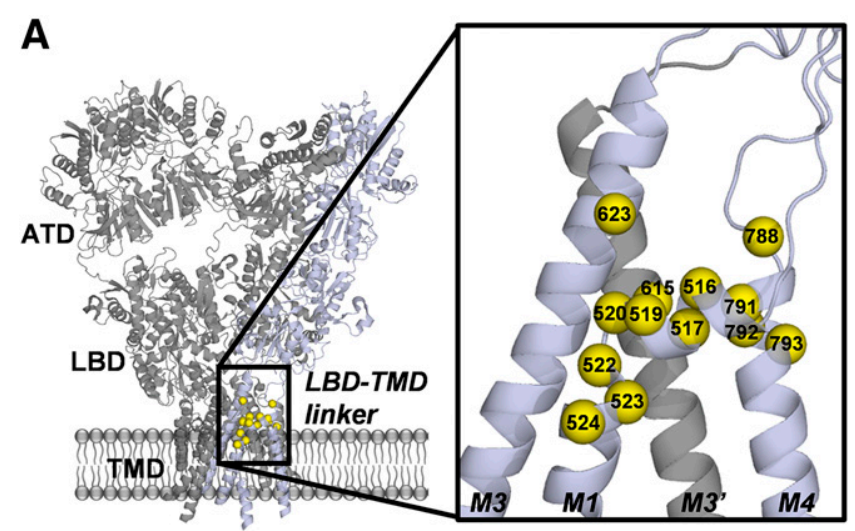

B

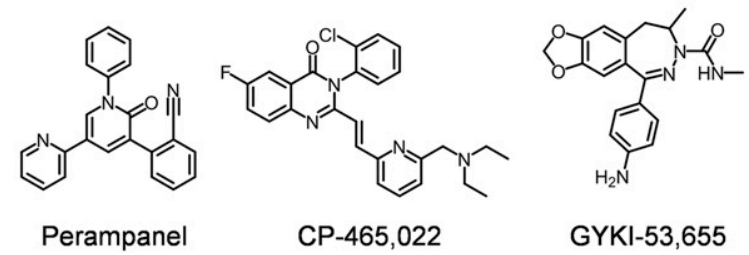

Fig. 1. Structure of the GluA2 AMPAR (PDB 3KG2) and the negative allosteric modulators. (A) Cartoon representation of the structure of the GluA2 receptor with indications of the major domain regions (ATD, LBD, and TMD). The LBD-TMD linker region is located in the rectangular box, which shows an expanded view of the linker region of a single subunit (light blue) including the M3 helix of a neighboring subunit (gray). Residue positions previously reported to affect NAM selectivity and/or potency are highlighted in yellow. (B) Chemical structures of the NAMs perampanel, CP-465,022, and GYKI-53,655.

surface. These results suggest that Gly substitutions at these positions may alter or completely disrupt receptor function. The Gly mutants for the remaining 11 positions displayed robust Glu-evoked $\mathrm{Ca}^{2+}$ signals that allowed determination of Glu $\mathrm{EC}_{50}$ values. For the majority of functional mutants, the Glu $\mathrm{EC}_{50}$ value was identical to or within the 3-fold range of the $\mathrm{EC}_{50}$ value for WT blac-GluA2 (Fig. 3; Table 1). For the 15 Gly substitutions in the S2-M4 and upper M4 segments, four mutants (L787G, V792G, F796G, and Y797G) were nonfunctional with intact cell surface expression (Supplemental Fig. 2; Supplemental Table 1), whereas Gly substitution at the remaining 11 positions yielded mutants with robust Gluevoked responses and $\mathrm{EC}_{50}$ values that were unchanged or within the 3-fold range of WT blac-GluA2 (Fig. 3B). Thus, in total, we generated 31 Gly mutants, of which 22 were functional and could be subjected to characterization of potential changes in GluA2 NAM pharmacology.

Effect of Glycine Substitutions on Inhibitory Potency of Perampanel, CP, and GYKI. We next used the $\left[\mathrm{Ca}^{2+}\right]_{\mathrm{i}}$ imaging assay to generate full concentrationinhibition relationships for PMP, CP, and GYKI (see Materials and Methods) to determine the $\mathrm{IC}_{50}$ value for each NAM across all functional Gly mutants (Table 2). Representative examples of concentration-response curves are shown in Fig 4. Fig 5 summarizes the relative changes in NAM potency produced by the Gly substitutions compared with WT blac-GluA2. Determination of specific $\mathrm{IC}_{50}$ values was not possible at some mutants that displayed decreased sensitivity to NAMs due to limitations in NAM solubility.

For the S1-M1 segment, we generally observed similar patterns of mutational effects for the three NAMs (Fig. 5). Specifically, removal of residue side chains at K511 and V514 
TABLE 1

Effect of mutations on GluA2 $\mathrm{EC}_{50}$ and $E_{\max }$ for glutamate The $\mathrm{EC}_{50}$ values were determined by the nonlinear fitting of composite concentration-inhibition data from at least three independent experiments (TEVC or $\mathrm{Ca}^{2+}$ ) (see Materials and Methods). Numbers in brackets denote the 95\% confidence interval. $* P<0.05 ; * * P<0.01 ; * * * P<0.001$ significantly different from blac-GluA2 (ANOVA). To maintain an overall familywise $\alpha$ of 5\%, for each dependent variable separate ANOVA with Dunnett's post hoc test were run at $\alpha=0.05 / \mathrm{dv}$, where $\mathrm{dv}$ is the number of dependent variables. N.D. not determined. N.F. nonfunctional.

\begin{tabular}{|c|c|c|c|}
\hline \multirow{2}{*}{ Construct } & \multirow{2}{*}{$\begin{array}{c}E_{\max }\left[\mathrm{Ca}^{2+}\right]_{\mathrm{i}}(\% \text { of WT }) \\
\%\end{array}$} & \multicolumn{2}{|c|}{$\mathrm{EC}_{50}$} \\
\hline & & $\underset{\mu M}{\left[\mathrm{Ca}^{2+}\right]_{\mathrm{i}}}$ & $\underset{\mu M}{\text { TEVC }}$ \\
\hline GluA2 & 100 & $17[16-17]^{* *}$ & 23 [23-23] \\
\hline blac-GluA2 & 102 [82-121] & 14 [14-15] & 27 [27-28] \\
\hline $\mathrm{K} 511 \mathrm{G}$ & $29[22-36]^{* * *}$ & $4.3[4.0-4.6]^{* *}$ & $30[30-31]^{*}$ \\
\hline $\mathrm{P} 512 \mathrm{G}$ & $134[91-177]$ & $27[26-29]^{* *}$ & $43[42-44]^{* *}$ \\
\hline V514G & $28[21-35]^{* * *}$ & $11[9.6-13]^{* *}$ & 26 [25-27] \\
\hline V514A & $36[17-56]^{* *}$ & $5.7[3.4-9.6]^{* *}$ & $12[12-12]^{* *}$ \\
\hline V514D & $76[24-128]$ & $3.7[2.2-6.3]^{* *}$ & $29[28-30]$ \\
\hline V514W & $63[42-84]$ & $7.5[6.6-8.6]^{* *}$ & $32[31-32]^{*}$ \\
\hline F515G & $86[61-112]$ & $38[37-39]^{* *}$ & $35[34-36]^{*}$ \\
\hline F515A & $135[-16$ to 286$]$ & $12[9.2-15]$ & N.D. \\
\hline S516G & $99[91-107]$ & $29[27-32]^{* *}$ & N.D. \\
\hline F517G & $38[32-44] * * *$ & $130[130-140]^{* * *}$ & N.D. \\
\hline F517A & $54[50-57]^{*}$ & $70[66-75]^{* * *}$ & N.D. \\
\hline L518G & N.F. & N.F. & N.D. \\
\hline L518A & $61[48-74]$ & $89[82-97]^{* * *}$ & $31[30-32]^{*}$ \\
\hline D519G & $63[41-86]$ & $8.9[8.0-9.8]^{* *}$ & N.D. \\
\hline D519N & $91[81-101]$ & 14 [14-15] & $37[37-38]^{* *}$ \\
\hline P520G & N.F. & N.F. & N.D. \\
\hline L521G & N.F. & N.F. & N.D. \\
\hline L521A & N.F. & N.F. & N.D. \\
\hline A522G & 99 [83-114] & $20[19-23]^{* *}$ & N.D. \\
\hline A522S & $63[47-80]^{*}$ & 13 [12-13] & N.D. \\
\hline Y523G & $93[73-113]$ & $28[26-30]^{* *}$ & N.D. \\
\hline Y523P & $87[81-94]$ & 12 [12-13] & N.D. \\
\hline Y523R & 99 [85-114] & $19[18-20]^{*}$ & N.D. \\
\hline E524G & $60[56-65]^{*}$ & $36[34-38]^{* *}$ & N.D. \\
\hline E524D & $47[23-71]^{* * *}$ & $19[17-21]^{*}$ & $37[37-38]^{* *}$ \\
\hline I525G & N.F. & N.F. & N.D. \\
\hline W526G & N.F. & N.F. & N.D. \\
\hline M527G & 99 [87-111] & $27[25-30]^{* *}$ & N.D. \\
\hline S615A & $68[-50$ to 187$]$ & $2.5[0.7-8.5]^{*}$ & $22[22-23]^{* *}$ \\
\hline $\mathrm{Y} 616 \mathrm{~F}$ & N.F. & N.F. & $26[26-26]$ \\
\hline F623A & 65 [39-91] & $90[79-100]^{* * *}$ & $16[16-17]^{* *}$ \\
\hline E782G & $104[87-120]$ & $33[31-35]^{* *}$ & N.D. \\
\hline K783G & 112 [100-125] & $11[10-12]^{* *}$ & N.D. \\
\hline $\mathrm{T} 784 \mathrm{G}$ & 143 [69-217] & $47[46-49]^{* * *}$ & 26 [26-27] \\
\hline S785G & 124 [108-140] & $49[46-51] * * *$ & $25[25-26]$ \\
\hline A786G & 100 [67-134] & $82[80-84] * * *$ & $36[34-38]^{* *}$ \\
\hline L787G & N.F. & N.F. & N.D. \\
\hline S788G & 111 [93-129] & 36 [34-39]** & N.D. \\
\hline L789G & $31[16-47] * * *$ & $55[52-58]^{* * * *}$ & N.D. \\
\hline S790G & 99 [73-125] & $21[19-22]^{*}$ & N.D. \\
\hline N791G & $90[76-105]$ & $24[22-25]^{* *}$ & N.D. \\
\hline N791A & 65 [23-108] & $6.0[5.0-7.2]^{* *}$ & $20[19-21]^{* *}$ \\
\hline N791F & $38[27-49]^{* * *}$ & $200[190-210]^{* * *}$ & N.D. \\
\hline N791L & $32[22-43] * * *$ & $8.9[7.5-10]^{*}$ & N.D. \\
\hline V792G & N.F. & N.F. & N.D. \\
\hline V792I & 77 [53-102] & 14 [13-16] & $33[32-33]^{* *}$ \\
\hline A793G & 81 [69-92] & $24[23-26]^{* *}$ & N.D. \\
\hline A793D & $49[36-62]^{* *}$ & $2.4[2.1-2.8]^{* *}$ & N.D. \\
\hline A793H & $69[51-88]$ & 13 [12-14] & N.D. \\
\hline G794D & 64 [49-79] & $5.2[4.6-5.7]^{* *}$ & $50[49-50]^{* * *}$ \\
\hline G794H & N.F. & N.F. & N.D. \\
\hline V795G & $72[48-96]$ & $32[30-35]^{* *}$ & N.D. \\
\hline F796G & N.F. & N.F. & N.D. \\
\hline Y797G & N.F. & N.F. & N.D. \\
\hline
\end{tabular}

produce loss-of-potency effects for PMP, CP, and GYKI, whereas removal at F517 produces gain-of-potency effects for all three NAMs (albeit only 3-fold for CP at F517G) (Fig. 5; Table 2). The similar effect patterns suggest that the S1-M1 linker is involved in a NAM binding or inhibitory mechanism, and furthermore corroborate the previous suggestions that PMP, CP, and GYKI have overlapping binding sites (Menniti et al., 2000; Hanada et al., 2011). For GYKI, additional changes in potency occur by removal of the F515 and D519 side chains (leading to gain of potency), and the S516 side chain (leading to loss of potency). These results indicate that GYKI might interact with additional residues in the S1-M1 region compared with PMP and CP. For the Gly mutations in the upper M1 segment (A522G to M527G), no substantial 
TABLE 2

$\mathrm{IC}_{50}$ values for PMP, $\mathrm{CP}$, and GYKI at WT and mutant GluA2 The $\mathrm{IC}_{50}$ values were determined by the nonlinear fitting of composite concentrationinhibition data from at least three independent experiments (TEVC or $\mathrm{Ca}^{2+}$ ) (see Materials and Methods). Numbers in brackets denote the 95\% confidence interval for the fitted $\mathrm{IC}_{50} *{ }^{*} P<0.05 ; * * P<0.01 ; * * P<0.001$ significantly different from blac-GluA2 (ANOVA). To maintain an overall familywise $\alpha$ of 5\%, for each dependent variable separate ANOVA with Dunnett's post hoc test were run at $\alpha=0.05 / \mathrm{dv}$, where dv is the number of dependent variables. N.D. not determined.

\begin{tabular}{|c|c|c|c|c|c|c|}
\hline \multirow[t]{2}{*}{ Construct } & \multicolumn{2}{|c|}{ PMP } & \multicolumn{2}{|c|}{$\mathrm{CP}$} & \multicolumn{2}{|c|}{ GYKI } \\
\hline & {$\left[\mathrm{Ca}^{2+}\right]_{\mathrm{i}}$} & TEVC & {$\left[\mathrm{Ca}^{2+}\right]_{\mathrm{i}}$} & TEVC & {$\left[\mathrm{Ca}^{2+}\right]_{\mathrm{i}}$} & TEVC \\
\hline GluA2 & $29[28-29]^{* * *}$ & $4.3[4.2-4.4]^{* * *}$ & $2.5[2.5-2.6]^{* *}$ & $2.5[2.4-2.6]$ & $230[230-230] * * *$ & 20 [19-21] \\
\hline blac-GluA2 & 16 [15-18] & $2.4[2.3-2.5]$ & $1.1[1.0-1.2]$ & $2.4[2.3-2.6]$ & 120 [110-120] & $25[25-26]$ \\
\hline $\mathrm{K} 511 \mathrm{G}$ & $>100 * * *$ & $9.6[9.3-9.9]^{* * *}$ & $9.3[7.0-12]^{* * *}$ & $3.3[3.1-3.4]$ & $>2000 * * *$ & $62[49-78]^{* * *}$ \\
\hline $\mathrm{P} 512 \mathrm{G}$ & $9.8[8.4-11]^{*}$ & $4.4[4.3-4.5]^{* * *}$ & $5.5[5.0-6.0]^{* * *}$ & $7.5[7.2-7.8]^{* * *}$ & $140[100-180]$ & $79[75-84]^{* * *}$ \\
\hline V514G & $>100 * * *$ & $29[26-31]^{* * *}$ & $>100 * * *$ & $10[9.6-10]^{* * *}$ & $>2000 * * *$ & $620[450-850]^{* * *}$ \\
\hline V514A & $>100 * * *$ & $4.7[4.4-4.9]^{* * *}$ & $27[23-31]^{* * *}$ & $3.3[3.1-3.6]$ & $>2000 * * *$ & $11[10-12]^{* * *}$ \\
\hline V514D & 19 [14-26] & $11[11-11]^{* * *}$ & $6.0[5.8-6.3]^{* * *}$ & $4.0[3.8-4.3]$ & $>2000 * * *$ & $69[66-73]^{* * *}$ \\
\hline V514W & $87[71-110]^{* * *}$ & $2.3[2.3-2.4]$ & $4.2[3.7-4.7] * * *$ & $0.9[0.9-1.0]^{* * *}$ & $>2000 * * *$ & 18 [18-19] \\
\hline F515G & $4.1[3.7-4.5]^{* * *}$ & $1.6[1.6-1.6]^{* * *}$ & $0.7[0.7-0.8]$ & $1.6[1.6-1.7]$ & $20[19-21]^{* * *}$ & $7.9[7.7-8.1]^{* * *}$ \\
\hline F515A & $4.5[3.7-5.6]^{* * *}$ & N.D. & $0.4[0.4-0.4]^{* *}$ & N.D. & $49[42-57]^{* * *}$ & N.D. \\
\hline S516G & 23 [19-27] & N.D. & $3.5[3.3-3.8]^{* * *}$ & N.D. & $670[520-860]^{* * *}$ & N.D. \\
\hline F517G & $1.0[1.0-1.1]^{* * *}$ & N.D. & $0.3[0.3-0.3]^{* * *}$ & N.D. & $13[12 \text { to } 13]^{* * *}$ & N.D. \\
\hline F517A & $4.9[4.6-5.2]^{* * *}$ & N.D. & $2.4[2.1-2.6]$ & N.D. & $53[47-58]^{* * *}$ & N.D. \\
\hline L518A & $2.9[2.5-3.2]^{* * *}$ & $3.8[3.7-3.9]^{* * *}$ & $0.8[0.8-0.8]$ & $1.9[1.8-1.9]$ & $28[27-30]^{* * *}$ & $28[27-29]$ \\
\hline D519G & $10[9.2-11]^{* *}$ & N.D. & $3.7[3.4-4.0]^{* * *}$ & N.D. & $19[17-20] * * *$ & N.D. \\
\hline D519N & $24[17-34]$ & $3.3[3.2-3.5]^{* * *}$ & $61[18-210]^{* * *}$ & $7.4[7.0-7.8]^{* * *}$ & $55[47-63]^{* * *}$ & $8.1[7.7-8.5]^{* * *}$ \\
\hline A522G & $17[14-20]$ & N.D. & $1.7[1.6-1.8]$ & N.D. & $310[280-350] * * *$ & N.D. \\
\hline A522S & $16[14-18]$ & N.D. & $2.4[2.2-2.7]$ & N.D. & $510[400-650] * * *$ & N.D. \\
\hline Y523G & $12[10-14]$ & N.D. & $1.2[1.2-1.3]$ & N.D. & 91 [77-1107] & N.D. \\
\hline Y523P & $18[12-27]$ & N.D. & $3.1[2.8-3.4]^{* *}$ & N.D. & $370[320-430]^{* * *}$ & N.D. \\
\hline Y523R & 15 [13-17] & N.D. & 1.8 [1.6-1.9] & N.D. & $84[76-93]^{*}$ & N.D. \\
\hline E524G & $9.5[8.7-10]^{* * *}$ & N.D. & $0.5[0.5-0.5]^{*}$ & N.D. & $48[43-52]^{* * *}$ & N.D. \\
\hline E524D & 23 [19-27] & $2.0[1.9-2.1]^{* * *}$ & $5.0[3.9-6.4]^{* * *}$ & $1.6[1.5-1.6]$ & $140[110-180]$ & $14[14-15]^{* *}$ \\
\hline M527G & $8.9[7.9-10]^{* *}$ & N.D. & $0.5[0.4-0.6]$ & N.D. & $120[100-140]$ & \\
\hline S615A & $>100 * * *$ & $2.2[2.1-2.3]$ & $3.5[3.3-3.7]^{*}$ & $0.2[0.2-0.2]^{* * *}$ & $>2000 * * *$ & $31[14-66]$ \\
\hline $\mathrm{Y} 616 \mathrm{~F}$ & N.D. & $0.9[0.5-1.7]^{* * *}$ & N.D. & $1.8[1.6-1.9]$ & N.D. & $26[15-46]$ \\
\hline F623A & $>100 * * *$ & $>100 * * *$ & $12[7.2-21]^{* * *}$ & $51[4.4-590]^{* * *}$ & $100[88-120]$ & $87[35-210]^{* * *}$ \\
\hline E782G & $12[11-13]$ & N.D. & $1.1[1.1-1.2]$ & N.D. & $100[97-110]$ & N.D. \\
\hline K783G & $27[19-39]^{* *}$ & N.D. & $2.7[2.6-2.8]^{*}$ & N.D. & $170[160-180]^{* *}$ & N.D. \\
\hline $\mathrm{T} 784 \mathrm{G}$ & $8.5[7.3-9.8]^{* * *}$ & $7.9[7.6-8.2]^{* * *}$ & $1.1[1.0-1.2]$ & $2.5[2.5-2.5]$ & $23[21-25]^{* * * *}$ & 22 [22-23] \\
\hline $\mathrm{S} 785 \mathrm{G}$ & $10[9.0-11]^{* *}$ & $3.5[3.4-3.7]^{* * *}$ & $1.1[1.0-1.2]$ & $1.7[1.6-1.7]$ & $39[36-42]^{* * *}$ & $12[12-12]^{* * *}$ \\
\hline A786G & $1.4[1.3-1.6]^{* * *}$ & $2.7[2.6-2.7]^{* *}$ & $0.6[0.5-0.6]$ & $3.1[3.0-3.2]$ & $18[17-19]^{* * *}$ & $11[11-12]^{* * *}$ \\
\hline L787A & $37[18-74]^{* * *}$ & N.D. & $7.1[6.6-7.6]^{* * *}$ & N.D. & $630[500-780]^{* * *}$ & N.D. \\
\hline S788G & $16[14-18]$ & N.D. & $0.6[0.6-0.6]$ & N.D. & $92[77-110]$ & N.D. \\
\hline L789G & $4.8[4.3-5.4]^{* * *}$ & N.D. & $0.4[0.3-0.4]^{* * *}$ & N.D. & $61[51-73]^{* * *}$ & N.D. \\
\hline S790G & $9.9[5.4-18]^{*}$ & N.D. & $1.5[1.4-1.7]$ & N.D. & 140 [100-180] & N.D. \\
\hline N791G & $8.7[6.4-12]^{* * *}$ & N.D. & $15[9.2-24] * * *$ & N.D. & $350[300-420]^{* * *}$ & N.D. \\
\hline N791A & $>100 * * *$ & $1.5[1.5-1.6]^{* * *}$ & $>100 * * *$ & $11[10-13]^{* * *}$ & $>2000^{* * *}$ & $21[21-22]$ \\
\hline $\mathrm{N} 791 \mathrm{~F}$ & $3.7[3.5-3.8]^{* * *}$ & N.D. & $1.9[1.9-2.0]$ & N.D. & $22[20-23]^{* * *}$ & N.D. \\
\hline N791L & $14[13-15]$ & N.D. & $25[19-33]^{* * *}$ & N.D. & $66[62-69]^{* * *}$ & N.D. \\
\hline V792I & $14[12-16]$ & $1.2[1.2-1.3]^{* * *}$ & $13[9.2-17]^{* * *}$ & $2.1[2.0-2.2]$ & $170[140-200]^{*}$ & $11[10-11]^{* * *}$ \\
\hline A793G & $12[8.9-17]$ & N.D. & $1.1[1.0-1.2]$ & N.D. & $180[150-220]^{* * *}$ & N.D. \\
\hline A793D & $27[18-41]^{* *}$ & N.D. & $170[8.6-3500]^{* * *}$ & N.D. & $190[170-200] * * *$ & N.D. \\
\hline A793H & 15 [12-18] & N.D. & $3.7[3.0-4.5]^{* * *}$ & N.D. & 110 [110-120] & N.D. \\
\hline G794D & $37[30-46]^{* * *}$ & $5.5[5.4-5.7]^{* * *}$ & $11[8.4-14]^{* * *}$ & $2.8[2.7-2.9]$ & $280[250-310] * * *$ & $40[39-41]^{*}$ \\
\hline V795G & 15 [10-21] & N.D. & $1.1[0.9-1.2]$ & N.D. & 87 [74-100] & N.D. \\
\hline
\end{tabular}

potency changes (here defined as more than a 5 -fold change in the $\mathrm{IC}_{50}$ value) were observed for any NAM (Fig. 5; Table 2); indicating that the upper M1 transmembrane domain may not be involved in NAM binding. However, notably, only four Gly mutants within the M1 region were characterized since the I525G and W526G mutants were not functional in the $\left[\mathrm{Ca}^{2+}\right]_{\mathrm{i}}$ assay and thus not amenable to pharmacological characterization.

For Gly mutants of the seven positions in the S2-M4 segment, A786G displayed a gain-of-potency effect for PMP and GYKI, whereas the remaining Gly substitutions did not substantially change the potency of any of the NAMs (Fig. 5). In the upper M4 segment, no substantial changes in NAM potencies were seen upon Gly substitutions from L789 to V795 (Fig. 5). The exception to this pattern is the effect of the N791G mutation on CP potency, for which removal of the asparagine side chain decreases the $\mathrm{IC}_{50}$ value of $\mathrm{CP}$ by more than 10 -fold, whereas PMP and GYKI potency does not substantially change.

In summary, we assessed the effects of Gly substitutions on NAM potency for 11 positions in the S1-M1/upper M1 segments and 11 positions in the S2-M4/upper M4 segments. Among these positions, six Gly substitutions in the S1-M1 segment displayed more than a 5 -fold change in $\mathrm{IC}_{50}$, whereas no effects were observed for the upper segment of the M1 transmembrane helix. Furthermore, one substitution in the S2-M4 region and one substitution in the upper M4 segment displayed substantial effects. Thus, overall, these results of the Gly scan implicate eight residues in potential formation of the NAM binding site in GluA2.

Mutational Analysis of Key NAM Binding Pocket Residues. To assess the role of side chains at the initial positions identified in the Gly scan (Fig. 5), we made additional mutants of potential key positions to introduce side chains with 
A
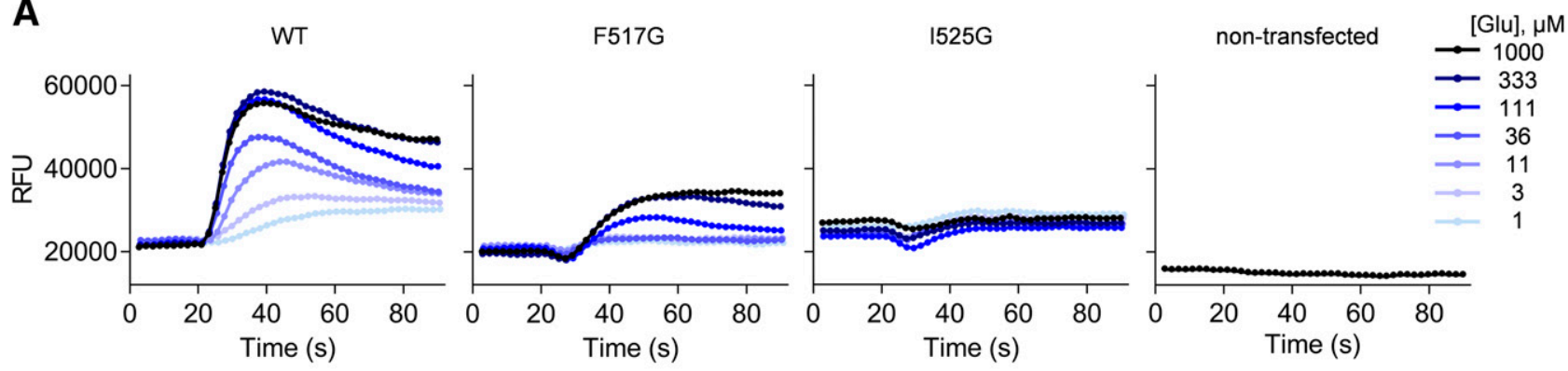

B
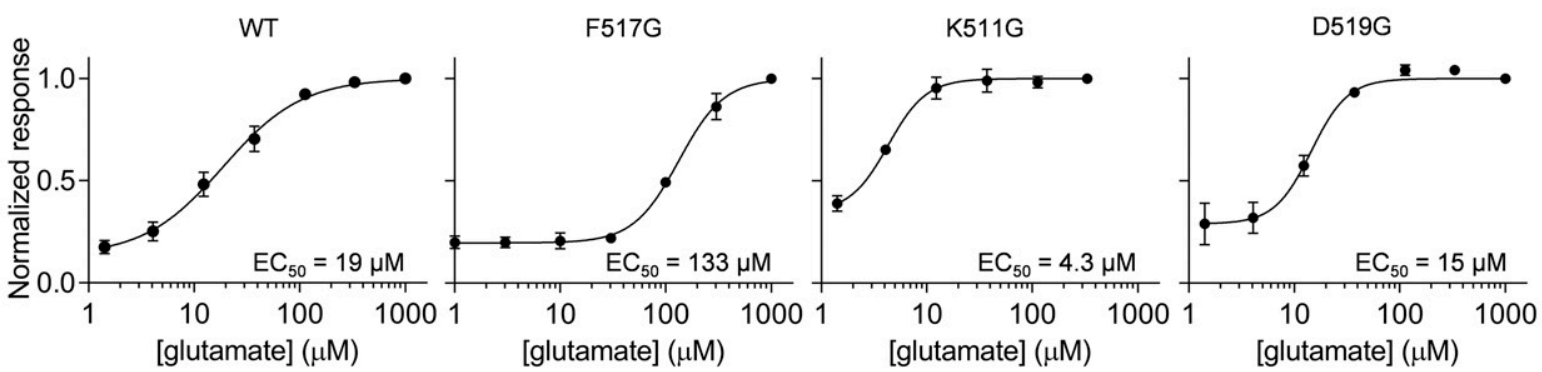

Fig. 2. Characterization of WT and mutant blac-GluA2 function in HEK cells using a 96-well-based $\left[\mathrm{Ca}^{2+}\right.$ ] imaging assay. (A) Representative traces from recordings of $\mathrm{Ca}^{2+}$ dye fluorescence from HEK293 cells transfected with WT and mutant blac-GluA2 receptors with the application of increasing Glu concentrations. F517G and I525G exemplify mutants with medium or no response, respectively, to Glu application. Traces represent mean fluorescence from four identical wells. (B) Representative examples of Glu concentration-response curves for WT, a loss-of-potency mutant (F517G), a gain-of-potency mutant (K511G), and a WT-like mutant (D519G). Data points represent the mean value from three independent concentration-response experiments (see Materials and Methods). Error bars are the S.D. and are shown when larger than symbol size.

different physicochemical properties (e.g., change the bulk size and/or hydrogen bonding properties of the side chain). Positions included mainly those where Gly substitution induced more than a 5-fold change in NAM potencies or was previously reported to change NAM activity (Balannik et al., 2005) (Fig. 5). At this point in the mutational analysis, X-ray crystallography structures were reported of homomeric GluA2 in complex with the same NAMs included in the present study (PMP, GYKI, and CP) (Yelshanskaya et al., 2016), showing that four nearly identical NAM binding sites are located within the LBD-TMD linker region of the tetramer. Although the electron densities around the identified NAM binding pockets in all of the GluA2/ NAM structures were insufficient to resolve several protein residue side chains, the structures provide unambiguous identification of the segments that form the binding pocket. Specifically, each NAM binding pocket is formed almost exclusively within each subunit by segments in the subunit S1-M1 linker (from K509 to P520, including the pre-M1 helix) and the extracellular facing regions of the M4 helix (from L787 to V795) and M3 helix (from Y616 to R628). The identification of these parts of the S1-M1 and upper M4 segments was in good agreement with the results from the Gly scan in that all of the eight positions identified to change NAM potency upon Gly substitution are contained herein (Fig. 5). Also, the GluA2/ NAM structures suggest that several residues within the upper M3 segment are in proximity to form direct interactions with the NAMs (Y616, L620, and F623, and S615 from the neighboring subunit). We included mutations of three of these residues to probe their potential involvement for direct interactions with the NAMs.

In total, we made additional mutations at 16 positions (Fig. 7) and determined effects on NAM potency using the $\left[\mathrm{Ca}^{2+}\right]_{\mathrm{i}}$ assay (Table 2 ). Also, we subjected selected mutants to determination of NAM potency using TEVC electrophysiology, which more directly measures AMPAR activation. Specifically, we expressed mutant and WT GluA2 receptors in Xenopus oocytes and determined the NAM $\mathrm{IC}_{50}$ values for inhibition of steady-state agonist-evoked currents (see Materials and Methods). The standard recording protocols are illustrated in Fig. 6. In general, the $\mathrm{IC}_{50}$ values obtained from the TEVC experiments were lower than those obtained from the $\left[\mathrm{Ca}^{2+}\right]_{\mathrm{i}}$ experiments (Table 2). For example, PMP and GYKI displayed $\mathrm{IC}_{50}$ values for WT GluA2 that were 5- and 10fold lower than the corresponding values obtained in the $\left[\mathrm{Ca}^{2+}\right]_{\mathrm{i}}$ experiments (Table 2). Additionally, relative changes in NAM potencies for mutants investigated by TEVC electrophysiology were generally smaller than the changes observed in the $\left[\mathrm{Ca}^{2+}\right]_{\mathrm{i}}$ assay. It should be noted that during agonist application in the $\left[\mathrm{Ca}^{2+}\right]_{\mathrm{i}}$ assay, desensitization is inhibited by coapplication of CTZ to produce robust $\mathrm{Ca}^{2+}$ responses (see Materials and Methods), whereas desensitization is not blocked during TEVC recordings. The difference in CTZ coapplication with NAM may underlie these differences since previous work has shown that CTZ, which binds in the LBD dimer interfaces to stabilize in a nondesensitizing conformation (Sun et al., 2002), allosterically influences the distinct NAM binding site to decrease apparent inhibitory potency (Johansen et al., 1995).

We created additional mutants at eight positions within the pre-M1 helix, the flanking S1-M1 linker segments, and the upper M1 and determined NAM potency (Fig. 7). At three positions in the pre-M1 helix (V514, F515, and F517), Gly substitutions indicated potential roles for NAM potency (Fig. 5). Since Gly has no side chain, Gly mutation is excellent for disrupting all potential ligand-protein interactions with the residue side chain. However, Gly also introduces flexibility to the protein backbone that might perturb local secondary structures or exert allosteric effects on the adjacent binding 
A
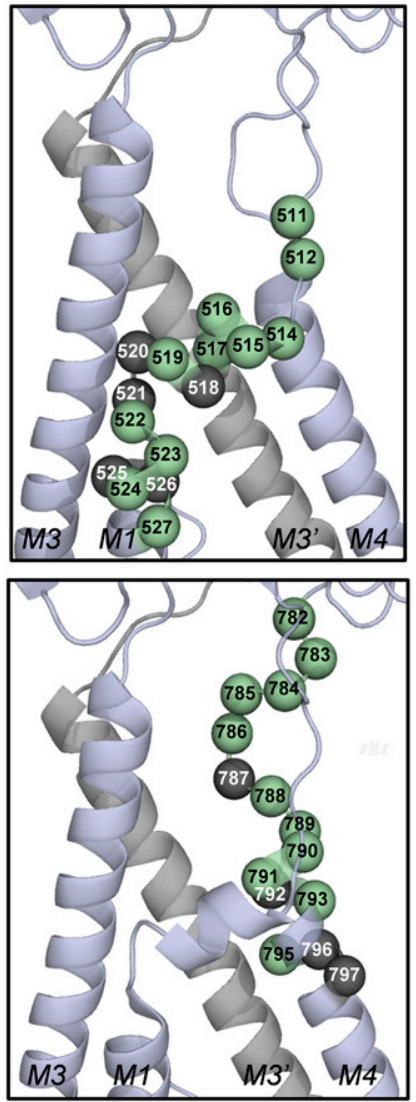

B
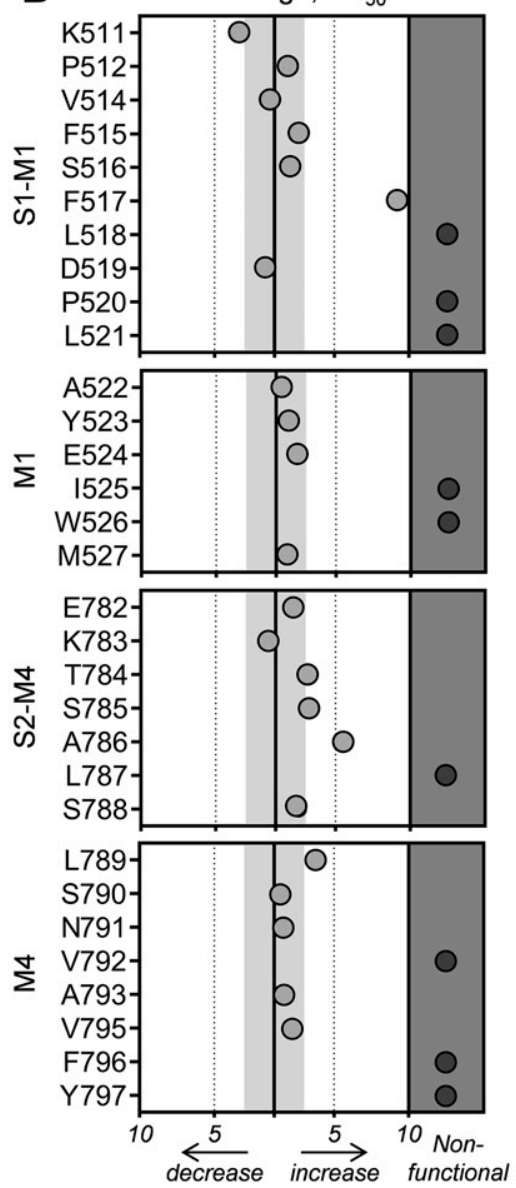

Fold change, $\mathrm{E}_{\max }$
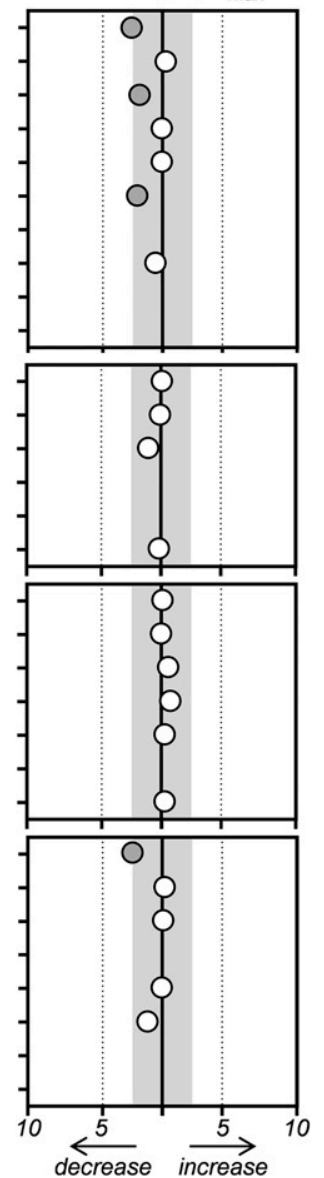

Fig. 3. Summary of functional characterization of Gly mutants of GluA2. (A) Overview of positions in GluA2 subjected to Gly substitution in the four segments of the LBD-TMD linker region of a single GluA2 subunit (S1-M1 linker, upper M1, S2-M4 linker, and upper M4; shown as cartoon representation in blue). M3 of the neighboring subunit is shown in gray cartoon representation. Mutated positions are shown as green spheres. Dark gray spheres indicate positions where Gly substitution rendered GluA2 nonfunctional. (B) Graphical summary of the fold change in Glu potency $\left(\mathrm{EC}_{50}\right)$ and maximal response amplitude $\left(E_{\max }\right)$ for mutants relative to WT blac-GluA2 measured by $\left[\mathrm{Ca}^{2+}\right]_{\mathrm{i}}$ imaging. Fold-change values for $\mathrm{EC}_{50}$ are calculated from the values in Table 1 as $\mathrm{EC}_{50 \text { (WT }} / \mathrm{EC}_{50 \mathrm{cmu}}$ tant) for mutants displaying decreased $\mathrm{EC}_{50}$ and as $\mathrm{EC}_{50 \text { (mutant }} / \mathrm{EC}_{50(\mathrm{WT})}$ for mutants displaying increased $\mathrm{EC}_{50}$. The light gray shaded area indicates mutants with fold changes less than 3fold compared with WT. The dark gray area indicates nonfunctional mutants. Filled gray circles indicate mutants that were statistically different from WT blac-GluA2 and white circles indicate mutants that were not statistically different from WT blac-GluA2 (see Table 1).

pocket structure. Therefore, we mutated V514, F515, and F517 to alanine (Ala) to reduce their hydrophobic side chains, but maintain backbone structural constraints. Also, for the L518 position, where Gly substitution destroyed measurable activity, we created the functional L518A mutant to probe the effect of shortening the hydrophobic side chain. In general, we considered performing Ala substitutions at all positions where Gly substitution produced nonfunctional receptors; in particular, for residues with large hydrophobic side chains (such as, for example, W526 in the M1 region and F796 and Y797 in the M4 region) to create potentially functional mutants that would enable characterization of the impact of side chain reduction on NAM potency. However, since GluA2/NAM structures were available at this point in the mutational analysis, we excluded residues that appeared to be outside the NAM binding pockets from further analysis under the assumption that further substitution of such residues was outside the primary aim of this part of the mutational analysis, which focused on probing potential direct ligand contacts. In agreement with previous observations (Yelshanskaya et al., 2016), Ala substitutions at F515, F517, and L518 generally produced no or minor changes in NAM potency. For F515 and F517, these results indicate that the observed changes by Gly substitutions may involve allosteric effects. In contrast, the V514A mutation produced reductions in potency for all NAMs that were similar to those produced by the V514G mutation (Fig. 7). To further explore V514, we mutated this residue to aspartate (Asp; introducing a negatively charged side chain) and tryptophan (Trp; increasing side chain size). Interestingly, the pattern of effects of these mutations differed among the NAMs, with GYKI potency being reduced at V514D and V514W, whereas PMP and CP were not affected or displayed smaller increases in $\mathrm{IC}_{50}$ values of up to 5-fold (Fig. 7). In the GluA2/NAM structures, V514 appears to be located outside the binding pocket (Fig. 7). Thus, the effect pattern of large decreases by Gly and Ala substitutions and less effect of Asp and Trp substitutions may not result from perturbation of direct interactions between the NAMs and the V514 side chain, but rather reflect a role of V514 in shaping the NAM binding site.

At position D519 in the pre-M1 region and A522, Y523, and E524 in the upper M1 region (Fig. 7), we made further mutations based on previous reports of combinations of mutations at these positions in GluA3 and GluK2 to change NAM potency (Balannik et al., 2005). D519N displayed decreased potency for $\mathrm{CP}$, which is in agreement with a suggested role of the $\gamma$-carboxylate in direct interaction with $\mathrm{CP}$ (Yelshanskaya et al., 2016). No other single mutations at A522, Y523, and E524 produced substantial effects on NAM potency (Fig. 7; Table 2). These results are in contrast to the previously suggested roles of these residues in interaction with GYKI (Balannik et al., 2005), but in general agreement with their positions outside the NAM binding pockets in the GluA2/NAM structures (Fig. 7).

We created additional mutants at five positions within the S2-M4 linker and upper M4 region (Fig. 7). The mutations were observed to produce similar effect patterns on PMP and 

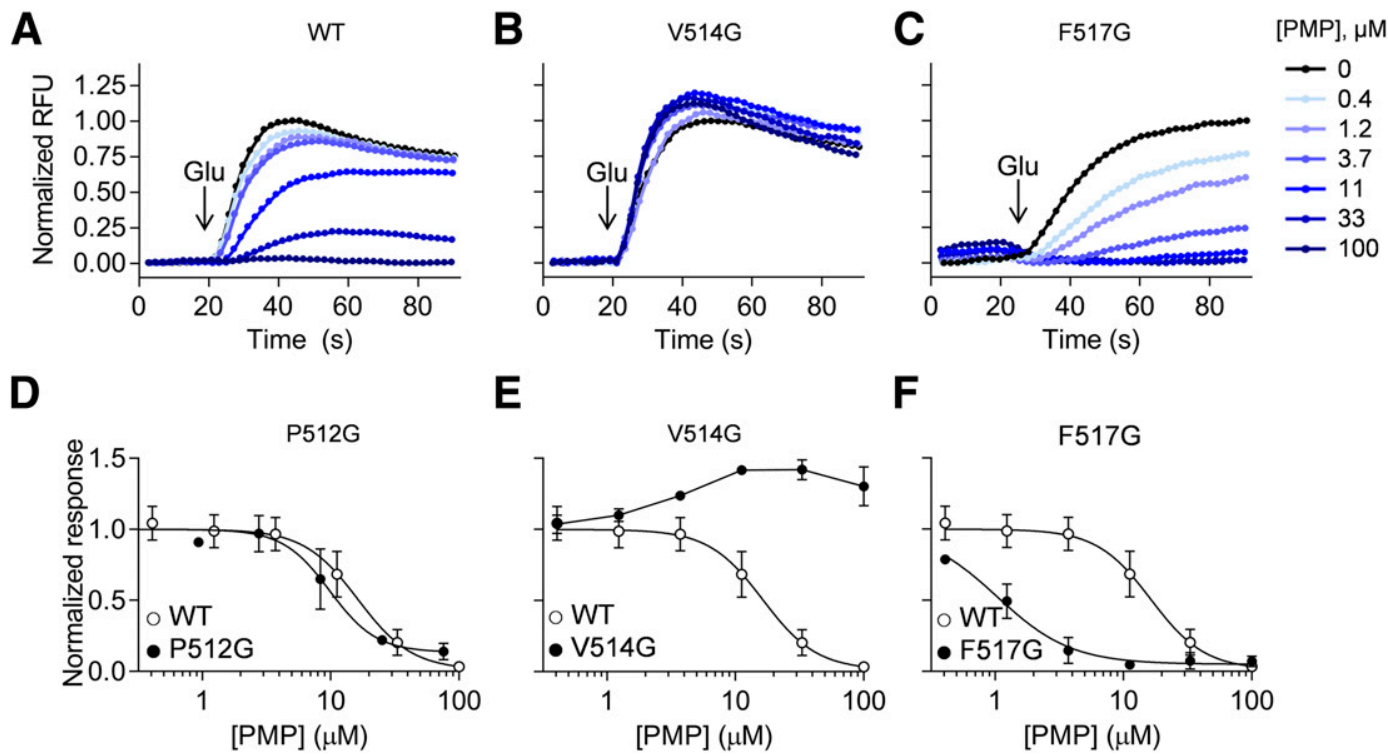

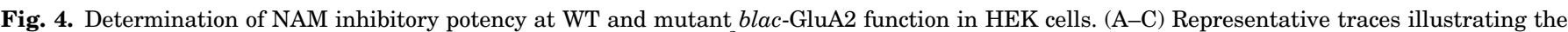

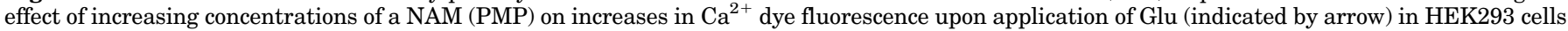

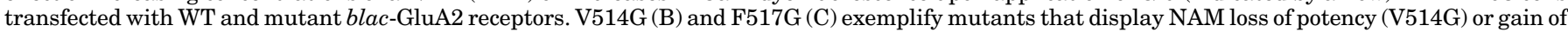

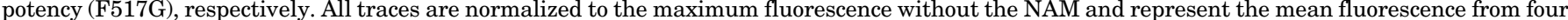

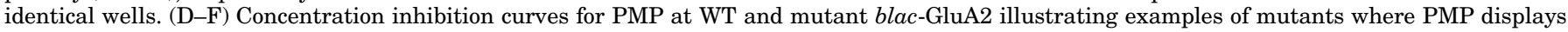

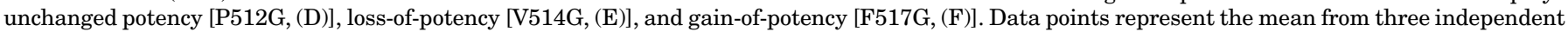
concentration-response experiments (see Materials and Methods). Error bars are the S.D. and are shown when larger than symbol size.

GYKI potency in the $\left[\mathrm{Ca}^{2+}\right]_{\mathrm{i}}$ assay (Fig. 7; Table 2), which both were sensitive to mutations at N791 and insensitive to mutations at V792, A793, and G794 (Fig. 7). In contrast, CP displayed sensitivity toward mutation at all positions (Fig. 7; Table 2), despite the side chains of positions 792-794 being located outside the NAM binding pocket. In the TEVC experiments, the effects on NAM potencies by mutations at V792, A793, and G794 were generally less than 3-fold, indicating that neither of these residues forms key interactions with the NAMs (Table 2).

In the M3 segment, we created three mutants (Fig. 7). The mutant Y616F probes the potential hydrogen bonding properties of the side chain hydroxyl for NAM binding. Y616F did not display sufficient activity in the $\left[\mathrm{Ca}^{2+}\right]_{\mathrm{i}}$ assay, but we were able to characterize the mutant using TEVC electrophysiology and found no substantial effect on NAM potency, suggesting that the side chain hydroxyl is not involved in direct ligand interactions. The mutant $\mathrm{S} 615 \mathrm{~A}$ also removed a side chain hydroxyl and displayed substantial loss of potency for PMP and GYKI in the $\left[\mathrm{Ca}^{2+}\right]_{\mathrm{i}}$ assay (Fig. 7; Table 2), in agreement with the previously reported effect of this mutation (Yelshanskaya et al., 2016). However, the S615A mutation did not change PMP and GYKI potencies measured by TEVC electrophysiology. Moreover, the mutant displayed a gain-of-potency effect for CP in TEVC, but not in the $\left[\mathrm{Ca}^{2+}\right]_{i}$ assay (Table 2). These results may indicate that the role of the S615 residue in NAM function differs depending on the desensitization state of the receptor, since desensitization was blocked by addition of CTZ in the $\left[\mathrm{Ca}^{2+}\right]_{\mathrm{i}}$ assay and the patch-clamp experiments performed by Yelshanskaya et al. (2016) but not in our TEVC experiments. To investigate the effect by addition of CTZ at the S615A mutant, we conducted TEVC recordings to determine NAM potencies at WT and S615A mutant receptors in the presence of CTZ (Supplemental Fig. 3; Supplemental Table 2). The presence of CTZ only caused minor (less than 3-fold) effects on NAM $\mathrm{IC}_{50}$ at WT GluA2 and blac-GluA2 receptors. However, in the presence of CTZ, the S615A mutant displayed substantial loss of potency for PMP and GYKI and no change in CP potency, similar to the results obtained in the $\left[\mathrm{Ca}^{2+}\right]_{\mathrm{i}}$ assay and the previously reported effect of the S615A mutation on PMP and GYKI potency (Yelshanskaya et al., 2016). These results indicate that blocking desensitization affects the role of $\mathrm{S} 615$ in NAM inhibition. F623A displayed substantial loss of potency for PMP and CP in both the $\left[\mathrm{Ca}^{2+}\right]_{\mathrm{i}}$ and TEVC assays, supporting a role of the side chain phenyl group in direct ligand interactions as previously suggested (Yelshanskaya et al., 2016).

Generation of NAM Binding Models in GluA2. The GluA2/NAM crystal structures have inadequate resolution in the NAM binding pockets for assignment of side chain conformations for many residues around the bound NAMs; therefore, they provide limited information on specific ligandprotein interactions. To address this issue, Yelshanskaya et al. (2016) modeled several side chains based on conformations observed in a higher resolution structure of GluA2 obtained in complex with the competitive antagonist ZK200775 (PDB code 3KG2) (Sobolevsky et al., 2009), and then used docking calculations to create models of NAM binding modes. However, the GluA2/ZK200775 structure also has several side chains missing in the NAM binding pocket; therefore, these were not modeled in the docking calculations (M. Kurnikova, personal communication). The missing side chains close to the NAM binding pocket include K508, K509, K511, E627, R628, E782, K783, and T784. Thus, the conformations of several side chains that might form critical interactions with PMP, CP, and GYKI remain unknown. Therefore, we repeated the docking with the inclusion of the missing side chain atoms in the binding sites using the GluA2/NAM structures as a template (see Materials and 
A
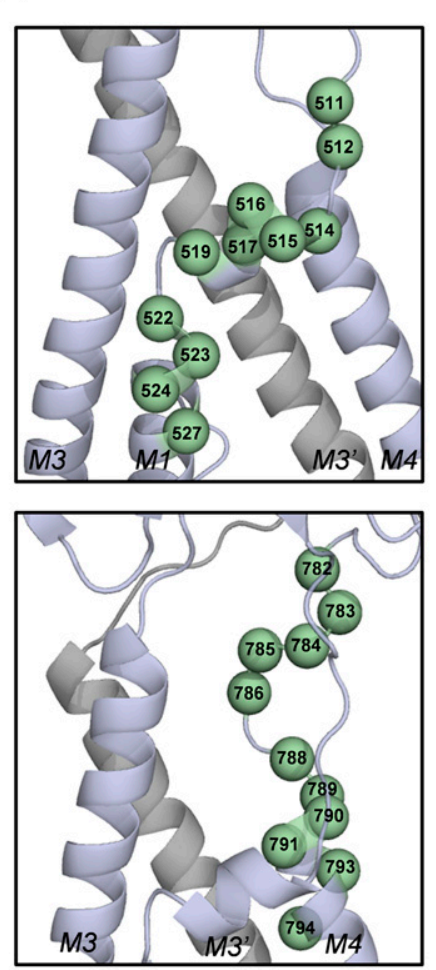

B
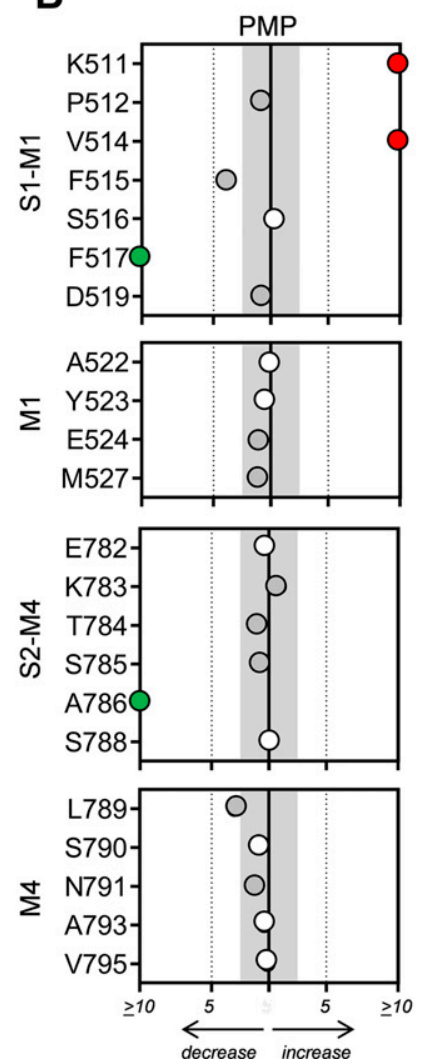

Fold change in $\mathrm{IC}_{50}$
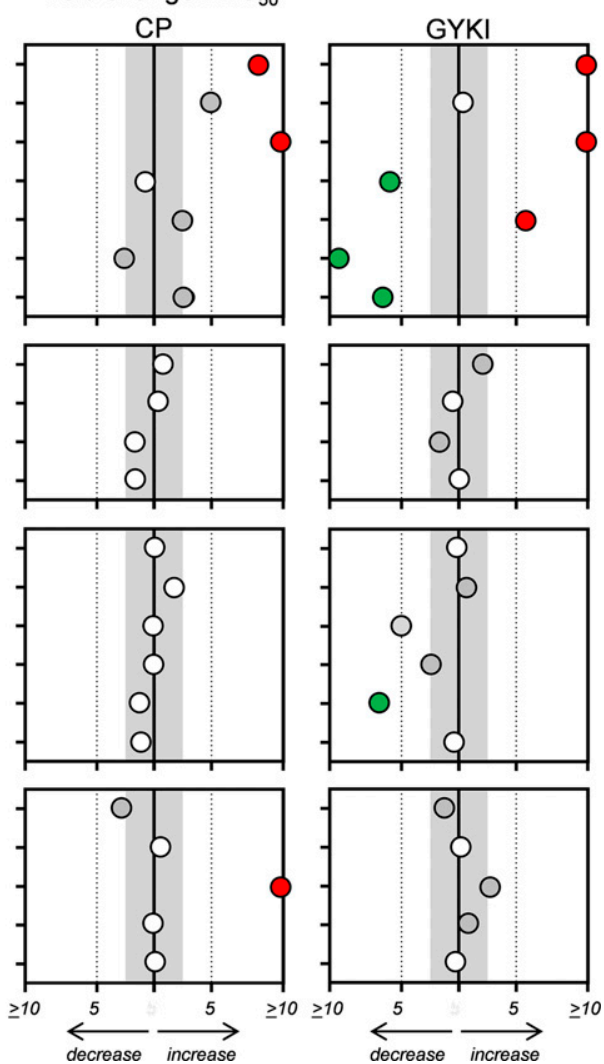

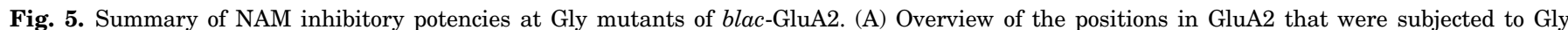

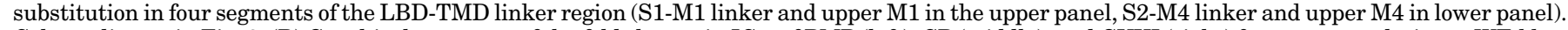

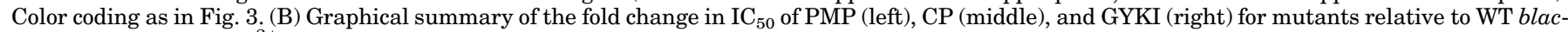

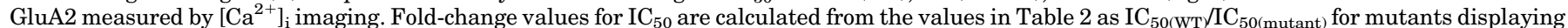

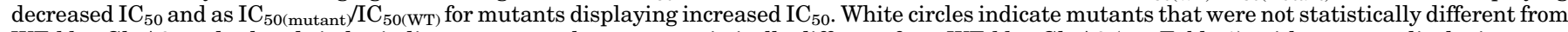

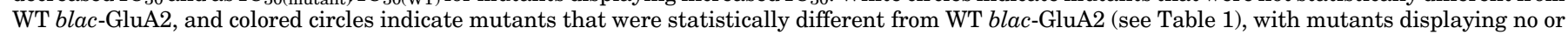

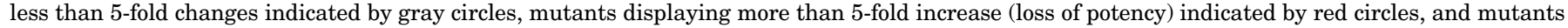
displaying more than 5-fold decrease (gain of potency) by green circles.

Methods). Initially, we employed a docking procedure with a flexible ligand docked into a rigid NAM binding pocket. To make the search as unbiased as possible, the search box used for the docking calculations was made large enough to include all four binding sites as well as the majority of the TMDs and the nearest part of the LBD. Of the 200 generated poses for each ligand (10 input conformations $\times 20$ poses per docking), at least $25 \%$ showed the ligand positioned in the binding site (27\% for PMP, 35\% for GYKI, and $79 \%$ for PMP). The remaining poses were located either in the pore, between transmembrane helices, or in between the TMDs and LBDs. The ligand poses in the binding sites showed considerable variability, possibly because only very few hydrogen bonds (HBs) were formed. However, we confirmed that poses similar to the binding modes observed in the crystal structures by Yelshanskaya et al. (2016) could be identified for each of the three ligands. The predicted binding energies for poses docked in the binding sites for all three ligands were similar, ranging from -7.4 to $-10.5 \mathrm{kcal} / \mathrm{mol}$; comparable to the results presented previously (Yelshanskaya et al., 2016). Thus, when docked into the GluA2/NAM structures with all side chains around the binding site added, all three NAM ligands can find binding modes very similar to those suggested by Yelshanskaya et al. (2016) (Supplemental Fig. 4). On this basis, we proceeded with the ligand orientations that were proposed from the GluA2/NAM structures to create three sets of binding models based on sampling flexible side chain orientations followed by energy minimizations to generate a total of 400 binding site models for each ligand (see Materials and Methods). Each model set represents a range of possible conformations of all binding pocket side chains and their potential ligand-protein interactions. Figure 8 illustrates the observed variability in side chain orientation for all residues within $5 \AA$ of the NAMs ( 26 residues in total). For all three NAMs, we found the majority of proposed protein-ligand interactions to be primarily hydrophobic. Specifically, among the 26 residues surrounding the NAMs, 14 were purely hydrophobic, and four were mostly hydrophobic with a single polar group (K509, K511, Y616, and W526). Notably, the positions of the hydrophobic side chains showed only very little variability among models. In contrast, the side chain of K511, which was not resolved in the GluA2/NAM X-ray crystal structures (Yelshanskaya et al., 2016), displayed a wide range of conformations with the K511 side chain pointing toward the NAM molecules in some models, and more toward the lipid bilayer region in others. Figure 9 shows a representative model for each NAM, representing frequently observed side chain orientations among the 400 models. In these representative models, the phenyl ring of PMP is stacked between F623 and P520 (Figs. 
A

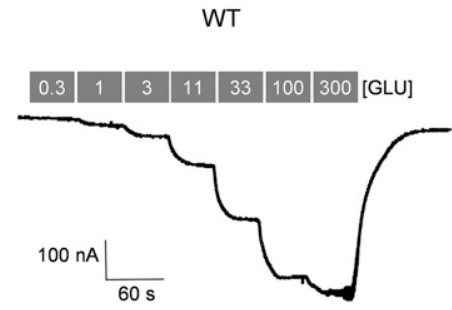

V514G

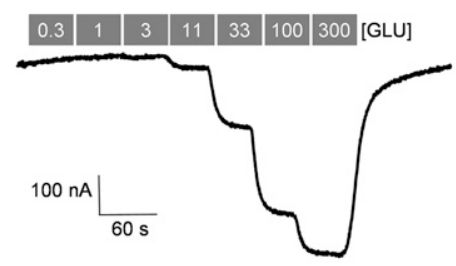

C

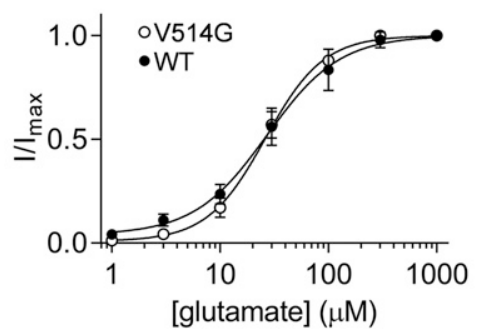

B
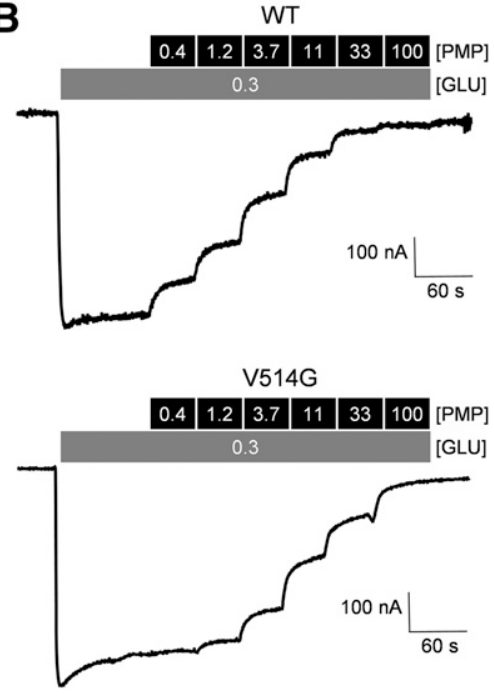

D

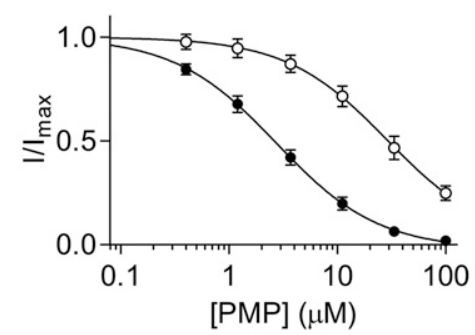

Fig. 6. TEVC characterization of WT and mutant blacGluA2 receptors. (A and B) Representative current traces illustrate the recording protocol used for concentrationresponse experiments for determination of $\mathrm{Glu}_{\mathrm{EC}} \mathrm{EC}_{50}(\mathrm{~A})$ and inhibitor $\mathrm{IC}_{50}(\mathrm{~B})$ at WT (upper traces) and representative mutant (V514G; lower traces) blac-GluA2 receptors expressed in Xenopus oocytes (see Materials and Methods). (C and D) Average composite concentration-response curves from four to eight oocytes. Error bars are the S.D. and are shown when larger than symbol size. The current responses are normalized to the maximal response produced by Glu (1 $\mathrm{mM})$.
8A and 9A); similar to the proposal by Yelshanskaya et al. (2016). F517 and V795 are lining the tip of the PMP pyridine ring, and the benzo-nitrile is lined by P512 and the carbon chain of K511. Similar to the X-ray crystal structures (Yelshanskaya et al., 2016), the methylene-dioxy group of GYKI is stacked against F623 (Figs. 8C and 9C). The aminophenyl group of GYKI is surrounded by a hydrophobic pocket consisting of F517, P520, L620, L624, and L787, and the 5methyl group is lined by P512 and the carbon chain of K511. The quinazolinone moiety of CP (surrounded by F517, P520, I611, L620, L787, V792, and V795) and the pyridine moiety are orientated parallel to F623 with its amino-substituent lined by the carbon chain of K511 and the cyano-phenyl pointing toward L787 and L624 (Figs. 8B and 9B).

In terms of potential hydrogen bond interactions, the NAM molecules may form HBs to both side chain or backbone atoms of the protein. Overall, 10 residues were observed within $5 \AA$ distances of the NAM ligands that have side chains containing HB donor or acceptor moieties (Figs. 8 and 9). Bulk analysis of the orientations of these side chains in the model sets showed five of these (S510 and K511 in the S1-M1 linker, S516 in the pre-M1 helix, S615 and Y616 in the upper M3 helix, and N791 in the upper M4 helix) to frequently be oriented to potentially form HB interactions to one or more of the NAM ligands with reasonable bond angles. It should be noted that the initial side chain orientations for the optimized models were generated using MODELLER (Sali and Blundell, 1993), which ensures that there is space for the ligands but does not take into account the chemical nature of the ligand. The models were subsequently energy minimized to optimize the protein-ligand interactions, using proper parameters for the ligands. However, minimization will only locate a nearby local minimum; therefore, if a side chain is not reasonably close to the ligand initially, it will not form a HB during minimization. Thus, the method may not allow for the identification of all possible HBs, and the frequency of a given $\mathrm{HB}$ is not necessarily a measure of the importance of that $\mathrm{HB}$ for the binding of the ligand. However, the method allowed us to identify several possible HBs that were not indicated in the crystal structures or through docking. With this in mind, the five residues (K511, S516, S615, Y616, and N791) can be considered as potential key HB interaction points for NAM binding (Supplemental Figs. 5-7). Of these, K511 has not previously been suggested as an interaction point since the side chain was not resolved or modeled in the GluA2/NAM X-ray crystal structures (Yelshanskaya et al., 2016). For PMP and GYKI, three side chains are implicated in potential HB interaction with the ligand (K511, S516, and N791 for PMP and S510, S615, Y616, and N791 for GYKI), whereas only N791 is suggested for CP (Fig. 9). The side chain of N791 is thus suggested to participate in HB interactions with all three NAMs, similar to the proposal by Yelshanskaya et al. (2016).

For PMP, the side chain most frequently observed in an orientation to form potential HBs was N791, through the interaction of the $\delta$-amide group of N791 with either the pyridine nitrogen or the nitrile of PMP (45\% of our models in total) (Supplemental Fig. 5). The $\gamma$-hydroxyl group of the S516 side chain was within the distance for HB formation with the nitrile of PMP as suggested by Yelshanskaya et al. (2016) in $28 \%$ of the models. The $\zeta$-ammonium group of the K511 side chain was less frequently ( $14 \%$ of the models) within the distance for interaction with the nitrile and/or the carbonyl 

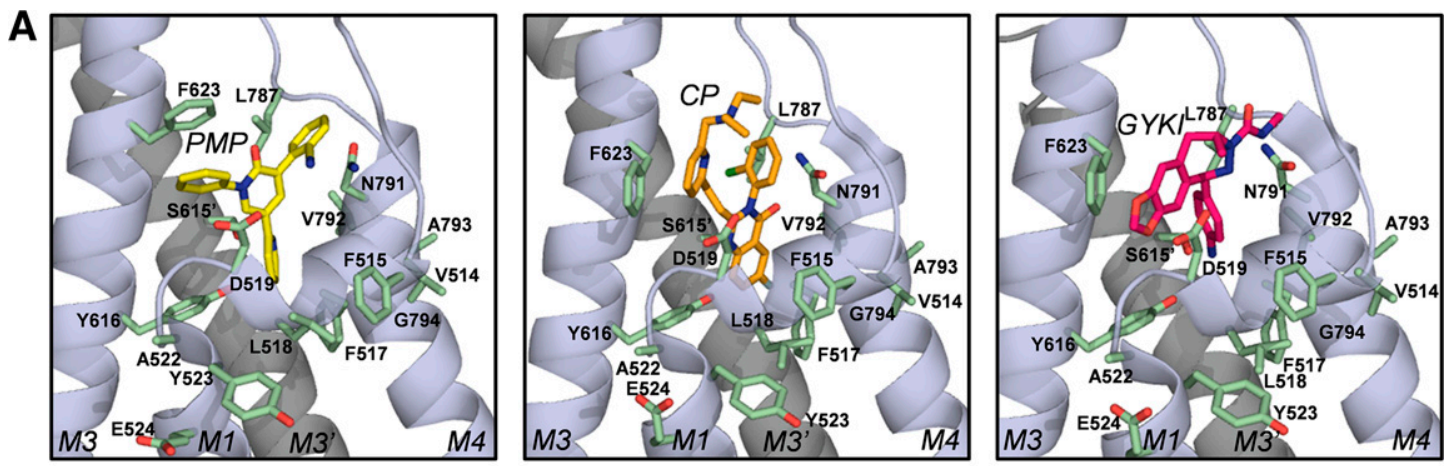

B

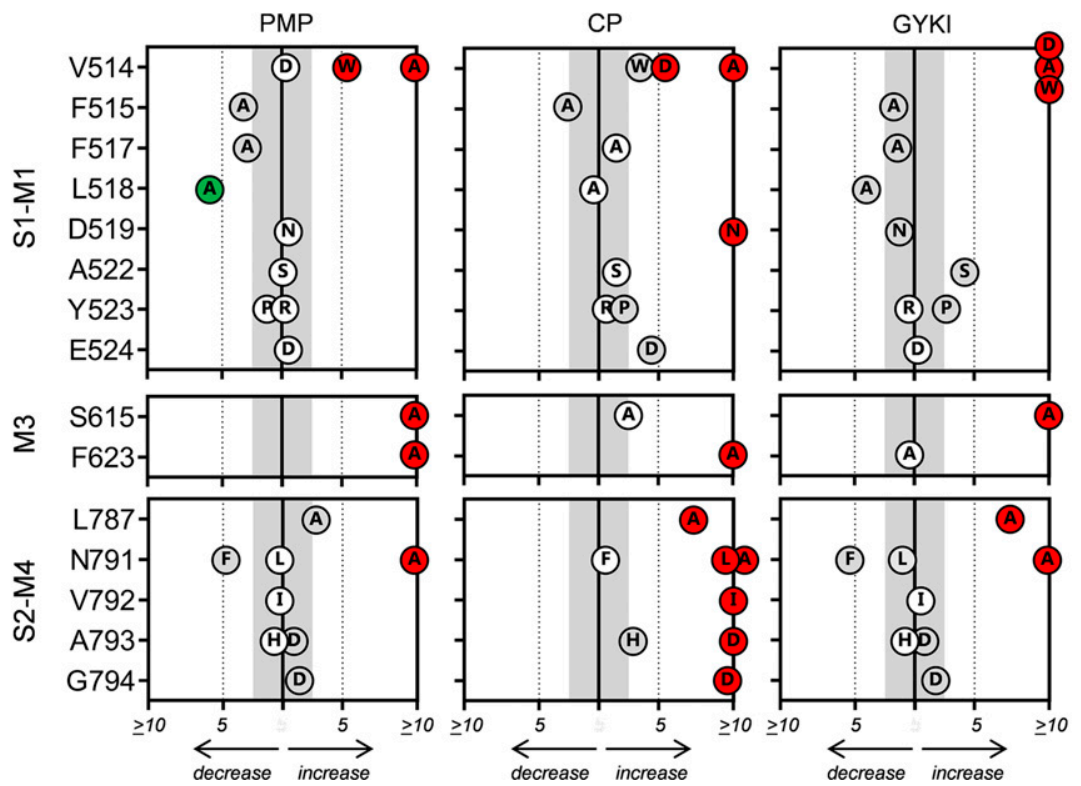

Fig. 7. Summary of effects on NAM inhibitory potency of non-Gly mutations in blac-GluA2. (A) Combined cartoon and stick representation of a single NAM binding site from the GluA2/NAM X-ray crystal structures (PDB 5L1F for PMP, PDB 5L1E for CP, and PDB 5L1H for GYKI) as determined by Yelshanskaya et al. (2016). Ligand positions and orientation of NAM structures are shown for PMP (left, yellow), CP (middle, orange), and GYKI (right, magenta). Residue positions subjected to non-Gly mutations are shown as green sticks. (B) Graphical summary of the fold change in $\mathrm{IC}_{50}$ measured by $\left[\mathrm{Ca}^{2+}\right]_{\mathrm{i}}$ imaging of PMP (left), CP (middle), and GYKI (right) for mutants relative to WT blac-GluA2. Fold change is calculated from the IC 50 values in Table 2 as $\mathrm{IC}_{50(\mathrm{WT}} / \mathrm{IC}_{50 \text { (mutant) }}$ for mutants displaying decreased $\mathrm{IC}_{50}$ and as $\mathrm{IC}_{50(\text { mutant }} / \mathrm{IC}_{50(\mathrm{WT})}$ for mutants displaying increased $\mathrm{IC}_{50}$. White circles indicate mutants that were not statistically different from WT blac-GluA2, and filled circles indicate mutants that were statistically different from WT blac-GluA2 (see Table 2), with mutants displaying no or less than 5-fold changes indicated by gray circles, mutants displaying more than 5-fold increase (loss of potency) indicated by red circles, and mutants displaying more than 5 -fold decrease (gain of potency) by the green circle.

groups of PMP. Potential HB interactions between PMP and the side chains of S615 or Y616 as suggested by Yelshanskaya et al. (2016) were generally not observed in our models. For GYKI, N791 was also frequently observed in an orientation to form potential HB interactions, through interaction of the $\delta$-amide group of N791 with the amide oxygen and/or the benzodiazepine nitrogen of GYKI ( $26 \%$ and $33 \%$ of the models, respectively) (Supplemental Fig. 7). Similar to the X-ray crystal structures (Yelshanskaya et al., 2016), the $\gamma$ - and $\eta$-hydroxyl groups of S615 and Y616 were within the distance for hydrogen bonding with the amine of GYKI in $72 \%$ and $75 \%$ of the models, respectively. In contrast to suggestions by Yelshanskaya et al. (2016), the $\gamma$-hydroxyl group of the S516 and S788 side chains was infrequently or not at all within the distance to form HB interactions with GYKI in our models. For $\mathrm{CP}$, the $\delta$-amide group of N791 was within the HB distance of the carbonyl of CP in 52\% of the models (Supplemental Fig. 6). Backbone-mediated HBs were not frequently observed in the models. Specifically, the analysis only suggests the backbone amide hydrogen of K511 to be oriented for potential HB interaction with the amide oxygen of GYKI (in $35 \%$ of the models) and the nitrile of PMP (in 24\% of the models) (Supplemental Figs. 5-7). In general, in combination with the GluA2/NAM structures, our models fit well with most of the key experimental observations from the mutational analysis and provide an additional framework for understanding molecular details of AMPAR interactions with NAMs of three different chemical classes.

\section{Discussion}

The molecular pharmacology of AMPARs involves multiple binding pockets targeted by many chemical classes of exogenous ligands acting as synthetic agonists, channel blockers, competitive antagonists, PAMs, and NAMs. The location, structure, and molecular properties of binding pockets for competitive antagonists (Sobolevsky et al., 2009), agonists (Dürr et al., 2014; Yelshanskaya et al., 2014; Twomey et al., 2017a; Zhu and Gouaux, 2017), and positive modulators (Dürr et al., 2014; Yelshanskaya et al., 2014; Twomey et al., 2017a; Zhu and 

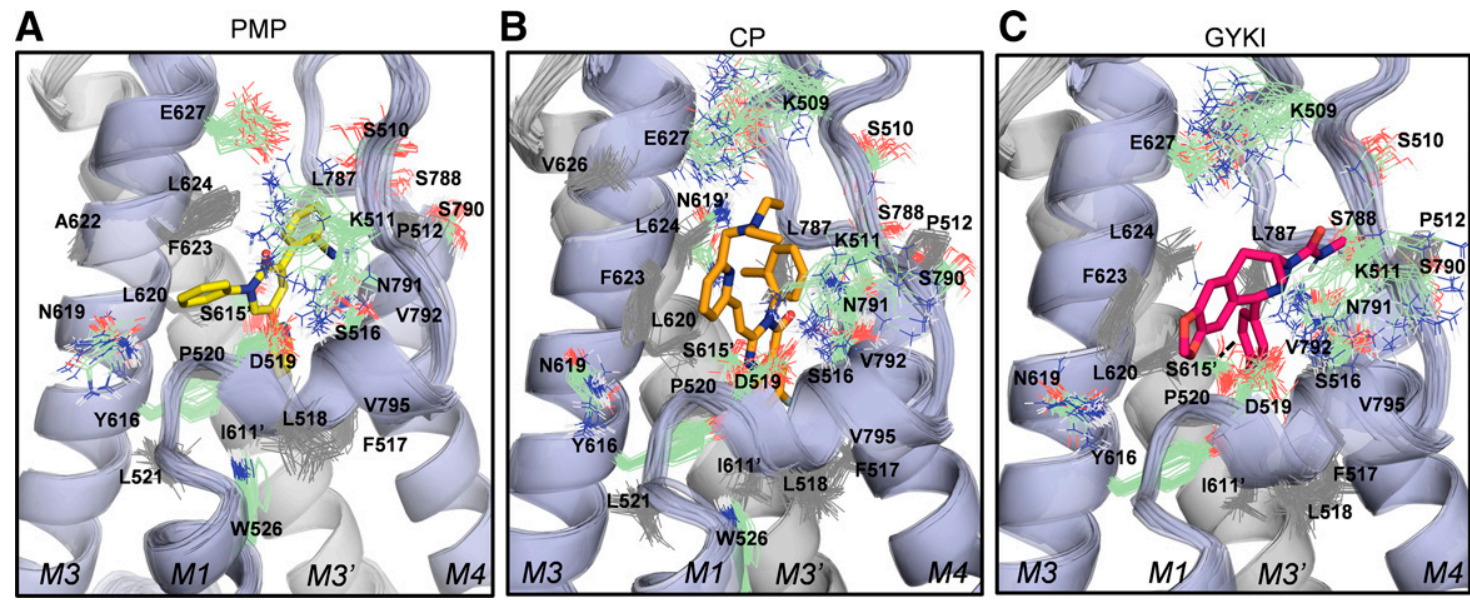

Fig. 8. Variability in side chain conformations among models of the GluA2 NAM binding pocket. (A-C) Overlay of 50 models for each GluA2 NAM binding site with residues within 5 A shown as lines, with hydrophobic residues in gray and hydrophilic residues in green. Each panel shows zoom-ins on the NAM binding pocket harboring PMP [(A) yellow], CP [(B) orange], and GYKI [(C) magenta].

Gouaux, 2017) are now defined at the atomistic level in the context of near full-length tetrameric AMPAR structures; leading to detailed models for how these ligands influence AMPAR dynamics to modulate function. In contrast, progress in NAM molecular pharmacology has been limited and essential aspects of NAM binding and functions have remained unknown. This is surprising since NAMs at present are the only class of AMPAR ligands from which a compound has emerged to become a therapeutic drug in humans. In the present study, our first aim was to use mutational analysis to pinpoint specific residues within the LBD-TMD linker regions as candidates for forming direct protein-ligand interactions. Our second aim was to use the mutational data to construct NAM binding models using existing GluA2 structures as docking templates. During work on both aims, X-ray crystallography structures of homomeric GluA2 in complex with the same prototypical NAMs selected for our study were reported (Yelshanskaya et al., 2016); thereby unambiguously identifying the number, location, and overall structure of the NAM binding pockets. However, the resolution of the X-ray structures was not sufficient for unambiguous identification of specific ligand-protein interactions involved in NAM binding. Thus, the data from our mutational analysis in combination with the recent GluA2/NAM structures form an improved basis for the construction of detailed binding mode models, which can be used for detailed analysis of ligand-protein interactions and serve as a framework for beginning to understand all molecular aspects of NAM function at AMPARs.

Overall, our mutational data fit well with the GluA2/NAM structures since most of the mutations changing NAM potencies were located in or near the NAM binding pockets, and these residues may thus potentially be critical for shaping the pocket or involved in direct ligand interactions. Some of these mutations concerned residues that were not resolved in the crystal structures. For example, we found that K511 in the S1M1 linker is important for the potency of all three NAMs (Fig. 5; Table 2). Mutational effects at K511 could not be interpreted from the existing GluA2/NAM structures since the side chain of K511 is not resolved or modeled (Yelshanskaya et al., 2016). Our results from the side chain optimizations
A

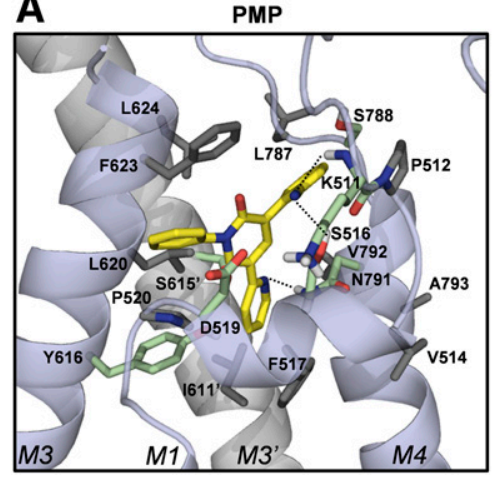

B

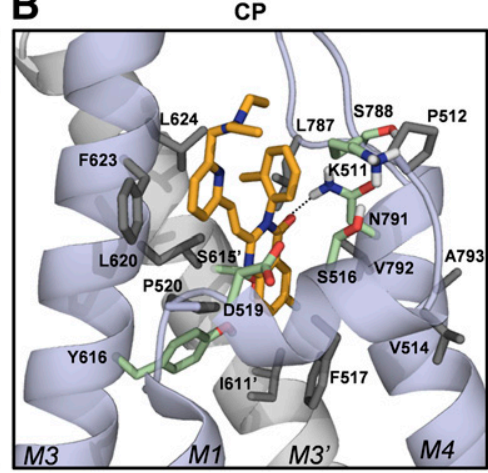

c

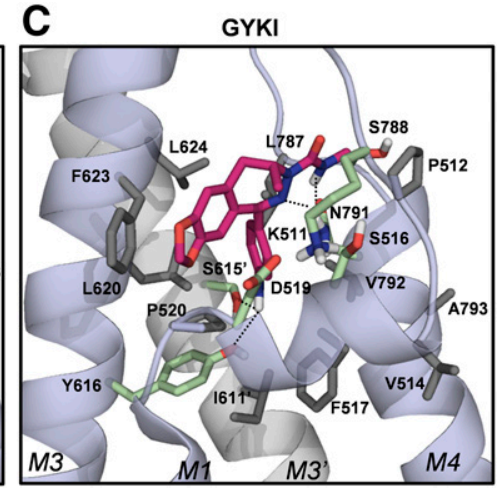

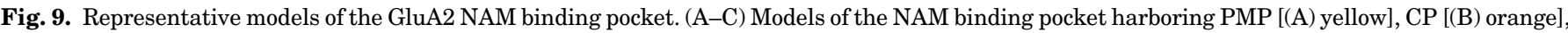

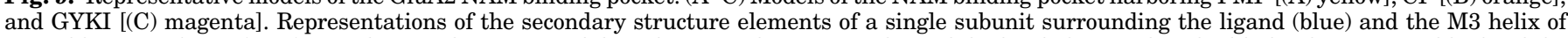

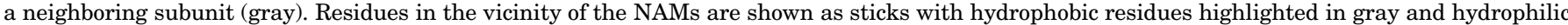

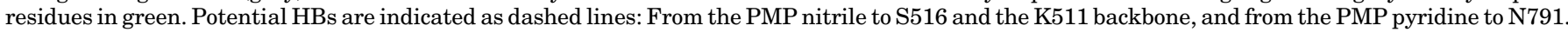

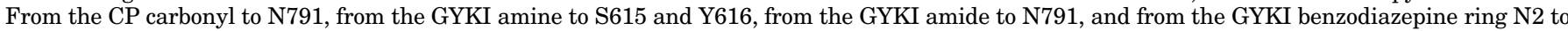
N791. 
suggest that K511 can adopt a position to form HBs with the NAM ligands, either by interaction with the side chain $\zeta$-ammonium group or the backbone hydrogen (Figs. 8 and 9). Also, the aliphatic carbons of the K511 side chain may line up against hydrophobic moieties of the NAMs, and thus form hydrophobic interactions. Interestingly, in other GluA2 structures for which the side chain of K511 has been resolved, several different conformations and orientations of the K511 side chain were observed. These included structures in which the side chain is orientated such that the primary amine points directly toward the empty NAM binding pocket (e.g., PDB codes $4 \mathrm{U} 4 \mathrm{~F}$ and $4 \mathrm{U} 4 \mathrm{G}$ ) (Yelshanskaya et al., 2014). In other structures, the K511 side chain points toward the membrane region; such as observed in a recently determined structure of a heteromeric GluA1/2 receptor in complex with transmembrane AMPAR regulatory protein $\gamma 8$ (PDB code 6QKC) (Herguedas et al., 2019). Interestingly, in this structure, the K511 side chain is pointing directly toward the extracellular region of $\gamma 8$ and a membrane lipid molecule. Hence, the variation in side chain conformations observed in the modeling appears convincing. Because we only tested the mutation of K511 to Gly, and Gly introduces backbone flexibility, it is plausible that the mutation may have affected hydrogen bonding capacities of both the backbone and side chain as well as hydrophobic interactions of the aliphatic carbons. Thus, our models and mutational data together point toward K511 as a novel interaction point for the NAMs. However, there are also examples of mutations of residues that we found exerted substantial effects on NAM potencies that cannot be readily explained by direct interactions in the current structures or our models. For example, V514 in the S1M1 linker region is in the periphery of the binding pocket, and its side chain is pointing away from the NAM ligands (Fig. 7). Yet, several mutations of this residue produced substantial changes in potency for all three NAMs (Figs. 5 and 7). V514 is located in the pre-M1 helix of the linker, and this residue potentially has a critical role for the stability or position of this structural element. The pre-M1 helix is part of the NAM pocket, and several residues in the pre-M1 helix make direct contacts to the NAMs. Thus, the results for V514 are likely an example of how point mutations can affect ligand potencies via indirect allosteric effects on ligand binding pockets.

Key interactions that were previously suggested from the GluA2/NAM crystal structures and mutational analysis by Yelshanskaya et al. (2016) are supported by our mutational data and models. These include hydrogen bonding interactions involving N791, and hydrophobic and $\pi$-stacking interactions involving P520 and F623 (Figs. 8 and 9). In contrast, the idea of key HB interactions of the side chains of S788 and Y616 with all of the tested NAMs is not supported by our mutational data or modeling. Specifically, in our modeling we only observed an HB interaction between Y616 and GYKI. However, the Y616F mutation did not change GYKI potency; therefore, the presence of this interaction was not supported (Fig. 7). S615 has also been suggested to form HBs to the NAM ligands, and mutation to Ala was previously reported to cause an order of magnitude loss of potency for GYKI and PMP (Yelshanskaya et al., 2016). When desensitization was blocked by CTZ in the $\left[\mathrm{Ca}^{2+}\right]_{\mathrm{i}}$ assay and TEVC experiments [similar to the patch-clamp electrophysiology experiments by Yelshanskaya et al. (2016)], we observed similar loss-of-potency effects by the S615A mutation, while CP potency remained unchanged (Fig. 7;
Supplemental Fig. 3). However, in the TEVC experiments without CTZ, S615A did not change potency for PMP and GYKI, but increased potency of CP by an order of magnitude. These results suggest that the role of $\mathrm{S} 615$ in NAM binding is state dependent, e.g., that block of desensitization by CTZ causes a different conformation of S615 compared with the receptor population in our TEVC experiments without CTZ in which the vast majority of receptors are desensitized. Interestingly, the S615A mutant has been previously reported to display slower rates of deactivation and desensitization and an increased fraction of steady-state to peak current (Yelshanskaya et al., 2017). Several other mutants display differential effects on NAM potencies between assays, albeit not to the same extent as the S615A mutant. Possibly, other residues in the NAM binding region play state-dependent roles in NAM inhibition due to statedependent variations in their structural conformations or mutational effects on receptor desensitization as reported for several residues in the region (Yelshanskaya et al., 2017). This aspect of NAM molecular pharmacology is of potential interest since AMPAR activation and desensitization are thought to involve transitions between multiple open and desensitized states (Robert et al., 2001; Jin et al., 2003; Robert and Howe, 2003). Also, the receptor activation mechanism may involve multiple preactivated states (Dürr et al., 2014). The dependence of NAM binding on different receptor conformations underlying this potential multitude of functional states and which state transitions are affected by NAM binding are at present unknown. Concerning this, it is interesting to note early observations (Johansen et al., 1995) that NAM potency appears to be affected by PAM ligands that now are known to bind in the LBD dimer interfaces and stabilize nondesensitized conformations of the LBD layer. Interestingly, our results indicate that the interactions of specific AMPAR residues with the NAMs can be altered by the presence of PAMs. Future modeling studies, potentially involving molecular dynamics simulations, aiming to compare the configuration of the NAM binding site in AMPAR structures representing different functional states seem warranted. In addition, inclusion of a lipid bilayer in the models may likely provide additional insight into how membrane lipids influence the NAM binding pocket. Such studies may reveal potentially important effects of the presence of different types of lipids on side chain orientation of residues such as V514, which in our models appears to orientate toward the membrane. Furthermore, an important open question to answer will be the role of cooperability of the four NAM sites per receptor complex; including the number of sites needed to be occupied to achieve inhibition of channel gating. Moreover, auxiliary subunits also influence AMPAR states and transitions; adding a layer of complexity to understanding the structural basis of AMPAR function (Dawe et al., 2016; Greger et al., 2017). In this relation, it is interesting to note that novel classes of NAMs have recently been reported that modulate AMPARs depending on the presence of certain members of the transmembrane AMPAR regulatory protein family (Gardinier et al., 2016; Maher et al., 2016; Azumaya et al., 2017).

In summary, we have performed mutational analysis and modeling of the NAM binding pockets and ligand binding modes in the GluA2 AMPAR, which in combination with recent X-ray crystal structures published by others (Yelshanskaya et al., 2016) constitute a step toward achieving a full molecular understanding of this important class of AMPAR inhibitors. 


\section{Acknowledgments}

We thank Alexander B. J. Jensen for excellent technical assistance.

\section{Authorship Contributions}

Participated in research design: Stenum-Berg, Musgaard, ChavezAbiega, Biggin, Kristensen.

Conducted experiments: Stenum-Berg, Musgaard, Chavez-Abiega, Thisted, Barella.

Contributed new reagents or analytic tools: Musgaard, Biggin.

Performed data analysis: Stenum-Berg, Musgaard, ChavezAbiega, Thisted, Barella, Kristensen.

Wrote or contributed to the writing of the manuscript: Stenum-

Berg, Musgaard, Biggin, Kristensen.

\section{References}

Azumaya CM, Days EL, Vinson PN, Stauffer S, Sulikowski G, Weaver CD, and Nakagawa T (2017) Screening for AMPA receptor auxiliary subunit specific modulators. PLoS One 12:e0174742.

Balannik V, Menniti FS, Paternain AV, Lerma J, and Stern-Bach Y (2005) Molecular mechanism of AMPA receptor noncompetitive antagonism. Neuron 48:279-288.

Berman HM, Westbrook J, Feng Z, Gilliland G, Bhat TN, Weissig H, Shindyalov IN, and Bourne PE (2000) The Protein Data Bank. Nucleic Acids Res 28:235-242.

Chen S, Zhao Y, Wang Y, Shekhar M, Tajkhorshid E, and Gouaux E (2017) Activation and desensitization mechanism of AMPA receptor-TARP complex by cryo-EM. Cell 170 (6):1234-1246.e14.

Chinea G, Padron G, Hooft RW, Sander C, and Vriend G (1995) The use of positionspecific rotamers in model building by homology. Proteins 23:415-421.

Citraro R, Aiello R, Franco V, De Sarro G, and Russo E (2014) Targeting $\alpha$-amino-3hydroxyl-5-methyl-4-isoxazole-propionate receptors in epilepsy. Expert Opin Ther Targets 18:319-334.

Cokić B and Stein V (2008) Stargazin modulates AMPA receptor antagonism. Neuropharmacology 54:1062-1070.

Dawe GB, Musgaard M, Aurousseau MRP, Nayeem N, Green T, Biggin PC, and Bowie D (2016) Distinct structural pathways coordinate the activation of AMPA receptor-auxiliary subunit complexes. Neuron 89:1264-1276.

Di Bonaventura C, Labate A, Maschio M, Meletti S, and Russo E (2017) AMPA receptors and perampanel behind selected epilepsies: current evidence and future perspectives. Expert Opin Pharmacother 18:1751-1764.

Dürr KL, Chen L, Stein RA, De Zorzi R, Folea IM, Walz T, Mchaourab HS, and Gouaux E (2014) Structure and dynamics of AMPA receptor GluA2 in resting, pre-open, and desensitized states. Cell 158:778-792.

Gardinier KM, Gernert DL, Porter WJ, Reel JK, Ornstein PL, Spinazze P, Stevens FC, Hahn P, Hollinshead SP, Mayhugh D, et al. (2016) Discovery of the first $\alpha$-amino-3-hydroxy-5-methyl-4-isoxazolepropionic acid (AMPA) receptor antagonist dependent upon transmembrane AMPA receptor regulatory protein (TARP) $\gamma-8$. J Med Chem 59:4753-4768.

Greger IH, Watson JF, and Cull-Candy SG (2017) Structural and functional architecture of AMPA-type glutamate receptors and their auxiliary proteins. Neuron 94:713-730.

Haering SC, Tapken D, Pahl S, and Hollmann M (2014) Auxiliary subunits: shepherding AMPA receptors to the plasma membrane. Membranes (Basel) 4:469-490.

Hanada T (2014) The discovery and development of perampanel for the treatment of epilepsy. Expert Opin Drug Discov 9:449-458.

Hanada T, Hashizume Y, Tokuhara N, Takenaka O, Kohmura N, Ogasawara A, Hatakeyama S, Ohgoh M, Ueno M, and Nishizawa Y (2011) Perampanel: a novel, orally active, noncompetitive AMPA-receptor antagonist that reduces seizure activity in rodent models of epilepsy. Epilepsia 52:1331-1340.

Herguedas B, Watson JF, Ho H, Cais O, García-Nafría J, and Greger IH (2019) Architecture of the heteromeric GluA1/2 AMPA receptor in complex with the auxiliary subunit TARP $\gamma 8$. Science 364:eaav9011.

Humphrey W, Dalke A, and Schulten K (1996) VMD: visual molecular dynamics. $J$ Mol Graph 14:33-38, 27-28.

Jackson AC and Nicoll RA (2011) The expanding social network of ionotropic glutamate receptors: TARPs and other transmembrane auxiliary subunits. Neuron 70:178-199.

Jespersen T, Grunnet M, Angelo K, Klaerke DA, and Olesen SP (2002) Dual-function vector for protein expression in both mammalian cells and Xenopus laevis oocytes. Biotechniques 32:536-538, 540.

Jin R, Banke TG, Mayer ML, Traynelis SF, and Gouaux E (2003) Structural basis for partial agonist action at ionotropic glutamate receptors. Nat Neurosci 6:803-810.

Johansen TH, Chaudhary A, and Verdoorn TA (1995) Interactions among GYKI52466, cyclothiazide, and aniracetam at recombinant AMPA and kainate receptors. Mol Pharmacol 48:946-955.

Joshi PR, Suryanarayanan A, and Schulte MK (2004) A vertical flow chamber for Xenopus oocyte electrophysiology and automated drug screening. Journal of neuroscience methods 132:69-79.

Karakas E, Regan MC, and Furukawa H (2015) Emerging structural insights into the function of ionotropic glutamate receptors. Trends Biochem Sci 40:328-337.

Kessels HW and Malinow R (2009) Synaptic AMPA receptor plasticity and behavior. Neuron 61:340-350.

Kumar J and Mayer ML (2013) Functional insights from glutamate receptor ion channel structures. Annu Rev Physiol 75:313-337.

Lam VM, Beerepoot P, Angers S, and Salahpour A (2013) A novel assay for measurement of membrane-protein surface expression using a $\beta$-lactamase. Traffic 14:778-784

Lazzaro JT, Paternain AV, Lerma J, Chenard BL, Ewing FE, Huang J, Welch WM, Ganong AH, and Menniti FS (2002) Functional characterization of CP-465,022, a selective, noncompetitive AMPA receptor antagonist. Neuropharmacology 42:143-153.
Liman ER, Tytgat J, and Hess P (1992) Subunit stoichiometry of a mammalian $\mathrm{K}^{+}$ channel determined by construction of multimeric cDNAs. Neuron 9:861-871.

Lindorff-Larsen K, Piana S, Palmo K, Maragakis P, Klepeis JL, Dror RO, and Shaw DE (2010) Improved side-chain torsion potentials for the Amber ff99SB protein force field. Proteins 78:1950-1958.

Maher MP, Wu N, Ravula S, Ameriks MK, Savall BM, Liu C, Lord B, Wyatt RM, Matta JA, Dugovic C, et al. (2016) Discovery and characterization of AMPA receptor modulators selective for TARP- $\gamma 8$. J Pharmacol Exp Ther 357:394-414.

Menniti FS, Chenard BL, Collins MB, Ducat MF, Elliott ML, Ewing FE, Huang JI, Kelly KA, Lazzaro JT, Pagnozzi MJ, et al. (2000) Characterization of the binding site for a novel class of noncompetitive $\alpha$-amino-3-hydroxy-5-methyl-4-isoxazolepropionic acid receptor antagonists. Mol Pharmacol 58:1310-1317.

Micale N, Colleoni S, Postorino G, Pellicanò A, Zappalà M, Lazzaro J, Diana V, Cagnotto A, Mennini T, and Grasso S (2008) Structure-activity study of 2,3-benzodiazepin-4-ones noncompetitive AMPAR antagonists: identification of the 1-(4amino-3-methylphenyl)-3,5-dihydro-7,8-ethylenedioxy-4H-2,3-benzodiazepin-4-one as neuroprotective agent. Bioorg Med Chem 16:2200-2211.

Mittapalli GK and Roberts E (2014) Structure activity relationships of novel antiepileptic drugs. Curr Med Chem 21:722-754.

Moncada C, Arvin B, Le Peillet E, and Meldrum BS (1991) Non-NMDA antagonists protect against kainate more than AMPA toxicity in the rat hippocampus. Neurosci Lett 133:287-290.

Poulsen MH, Lucas S, Bach TB, Barslund AF, Wenzler C, Jensen CB, Kristensen AS, and Strømgaard K (2013) Structure-activity relationship studies of argiotoxins: selective and potent inhibitors of ionotropic glutamate receptors. J Med Chem 56:1171-1181.

Robert A and Howe JR (2003) How AMPA receptor desensitization depends on receptor occupancy. J Neurosci 23:847-858

Robert A, Irizarry SN, Hughes TE, and Howe JR (2001) Subunit interactions and AMPA receptor desensitization. J Neurosci 21:5574-5586.

Sali A and Blundell TL (1993) Comparative protein modelling by satisfaction of spatial restraints. J Mol Biol 234:779-815

Salomon-Ferrer R, Case DA, and Walker RC (2013) An overview of the Amber biomolecular simulation package. Wires Comput Mol Sci 3:198-210.

Sobolevsky AI, Rosconi MP, and Gouaux E (2009) X-ray structure, symmetry and mechanism of an AMPA-subtype glutamate receptor. Nature 462:745-756.

Sólyom S and Tarnawa I (2002) Non-competitive AMPA antagonists of 2,3-benzodiazepine type. Curr Pharm Des 8:913-939.

Sørensen L, Strømgaard K, and Kristensen AS (2014) Characterization of intracellular regions in the human serotonin transporter for phosphorylation sites. ACS Chem Biol 9:935-944.

Sousa da Silva AW and Vranken WF (2012) ACPYPE-AnteChamber PYthon Parser interfacE. BMC Res Notes 5:367.

Sun Y, Olson R, Horning M, Armstrong N, Mayer M, and Gouaux E (2002) Mechanism of glutamate receptor desensitization. Nature 417:245-253.

Traynelis SF, Wollmuth LP, McBain CJ, Menniti FS, Vance KM, Ogden KK, Hansen KB, Yuan H, Myers SJ, and Dingledine R (2010) Glutamate receptor ion channels: structure, regulation, and function. Pharmacol Rev 62:405-496.

Trott O and Olson AJ (2010) AutoDock Vina: improving the speed and accuracy of docking with a new scoring function, efficient optimization, and multithreading. $J$ Comput Chem 31:455-461.

Twomey EC and Sobolevsky AI (2018) Structural mechanisms of gating in ionotropic glutamate receptors. Biochemistry 57:267-276.

Twomey EC, Yelshanskaya MV, Grassucci RA, Frank J, and Sobolevsky AI (2016) Elucidation of AMPA receptor-stargazin complexes by cryo-electron microscopy. Science 353:83-86.

Twomey EC, Yelshanskaya MV, Grassucci RA, Frank J, and Sobolevsky AI (2017a) Channel opening and gating mechanism in AMPA-subtype glutamate receptors. Nature 549:60-65.

Twomey EC, Yelshanskaya MV, Grassucci RA, Frank J, and Sobolevsky AI (2017b) Structural bases of desensitization in AMPA receptor-auxiliary subunit complexes. Neuron 94 (3):569-580.e5.

Van Der Spoel D, Lindahl E, Hess B, Groenhof G, Mark AE, and Berendsen HJC (2005) GROMACS: fast, flexible, and free. J Comput Chem 26:1701-1718.

Vriend G (1990) WHAT IF: a molecular modeling and drug design program. $J$ Mol Graph 8 (1):52-56, 29.

Wang J, Wolf RM, Caldwell JW, Kollman PA, and Case DA (2004) Development and testing of a general amber force field. J Comput Chem 25:1157-1174.

Yelshanskaya MV, Li M, and Sobolevsky AI (2014) Structure of an agonist-bound ionotropic glutamate receptor. Science 345:1070-1074.

Yelshanskaya MV, Mesbahi-Vasey S, Kurnikova MG, and Sobolevsky AI (2017) Role of the ion channel extracellular collar in AMPA receptor gating. Sci Rep 7:1050.

Yelshanskaya MV, Singh AK, Sampson JM, Narangoda C, Kurnikova M, and Sobolevsky AI (2016) Structural bases of noncompetitive inhibition of AMPA-subtype ionotropic glutamate receptors by antiepileptic drugs. Neuron 91:1305-1315.

Zarate CA Jr and Manji HK (2008) The role of AMPA receptor modulation in the treatment of neuropsychiatric diseases. Exp Neurol 211:7-10

Zhao Y, Chen S, Swensen AC, Qian WJ, and Gouaux E (2019) Architecture and subunit arrangement of native AMPA receptors elucidated by cryo-EM. Science 364:355-362.

Zhu S and Gouaux E (2017) Structure and symmetry inform gating principles of ionotropic glutamate receptors. Neuropharmacology 112 (Pt A):11-15.

Zwart R, Sher E, Ping X, Jin X, Sims JR, Chappell AS, Gleason SD, Hahn PJ, Gardinier K, Gernert D, et al. (2014) Perampanel, an antagonist of $\alpha$-amino-3hydroxy-5-methyl-4-isoxazolepropionic acid receptors, for the treatment of epilepsy: studies in human epileptic brain and nonepileptic brain and in rodent models. J Pharmacol Exp Ther 351:124-133.

Address correspondence to: Anders S. Kristensen, Department of Drug Design and Pharmacology, University of Copenhagen, Universitetsparken 2 DK-2100 Copenhagen, Denmark. E-mail: ask@sund.ku.dk 